\title{
Hybrid-Liouvillian formalism connecting exceptional points of non-Hermitian Hamiltonians and Liouvillians via postselection of quantum trajectories
}

\author{
Fabrizio Minganti, ${ }^{1, *}$ Adam Miranowicz, ${ }^{1,2, \dagger}$ Ravindra W. \\ Chhajlany, ${ }^{1,2, \ddagger}$ Ievgen I. Arkhipov ${ }^{3, \S}$ and Franco Nori ${ }^{1,4,}$ ף \\ ${ }^{1}$ Theoretical Quantum Physics Laboratory, RIKEN Cluster for Pioneering Research, Wako-shi, Saitama 351-0198, Japan \\ ${ }^{2}$ Faculty of Physics, Adam Mickiewicz University, PL-61-614 Poznań, Poland \\ ${ }^{3}$ Joint Laboratory of Optics of Palacký University and Institute of Physics of \\ CAS, Faculty of Science, Palacký University, 77146 Olomouc, Czech Republic \\ ${ }^{4}$ Physics Department, The University of Michigan, Ann Arbor, Michigan 48109-1040, USA
}

(Dated: June 26, 2020)

\begin{abstract}
Exceptional points (EPs) are degeneracies of classical and quantum open systems, which are studied in many areas of physics including optics, optoelectronics, plasmonics, and condensed matter physics. In the semiclassical regime, open systems can be described by phenomenological effective non-Hermitian Hamiltonians (NHHs) capturing the effects of gain and loss in terms of imaginary fields. The EPs that characterize the spectra of such Hamiltonians (HEPs) describe the time evolution of a system without quantum jumps. It is well known that a full quantum treatment describing more generic dynamics must crucially take into account such quantum jumps. In a recent paper [F. Minganti et al., Phys. Rev. A 100, 062131 (2019)],we generalized the notion of EPs to the spectra of Liouvillian superoperators governing open system dynamics described by Lindblad master equations. Intriguingly, we found that in situations where a classical-to-quantum correspondence exists, the two types of dynamics can yield different EPs. In a recent experimental work [M. Naghiloo et al. Nat. Phys. 15, 1232 (2019)], it was shown that one can engineer a non-Hermitian Hamiltonian in the quantum limit by postselecting on certain quantum jump trajectories. This raises an interesting question concerning the relation between Hamiltonian and Lindbladian EPs, and quantum trajectories. We discuss these connections by introducing a hybrid-Liouvillian superoperator, capable of describing the passage from an $\mathrm{NHH}$ (when one postselects only those trajectories without quantum jumps) to a true Liouvillian including quantum jumps (without postselection). Beyond its fundamental interest, our approach allows to intuitively relate the effects of postselection and finite-efficiency detectors.
\end{abstract}

\section{CONTENTS}

I. Introduction

II. Liouvillians and Quantum Trajectories
A. Liouvillian spectrum, Hamiltonian and Liouvillian EPs
B. Quantum trajectories
C. Postselection of quantum trajectories

III. Hybrid-Liouvillian formalism and its connection to postselection
A. Interpretation of the hybrid Liouvillian in terms of post-selected trajectories
B. Postselected quantum trajectory and inefficient detectors
C. The $q>1$ case

IV. Example 1: a model with a LEP without HEPs 7

A. Equivalence between postselection trajectories and hybrid Liouvillian

\section{7}

1. Two-detector post-selected trajectories

2. Imperfect detection

B. Transition of $\mathrm{NHH}$ to $\mathcal{L}$ and the appearance of a LEP

3

4

5

5

5

6

7

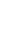

V. Example 2 of a model with inequivalent LEP and HEP

A. Transition from the HEP to the LEP

VI. Conclusions

Acknowledgments

A. Eigenvalues and eigenmatrices of the hybrid Liouvillian in Eq. (41)

1. Eigenvalues $\lambda_{i}$

2. Eigenmatrices $\hat{\rho}_{i}$

References

\footnotetext{
* fabrizio.minganti@riken.jp

$\dagger$ adam@riken.jp

‡ ravi@amu.edu.pl

§ ievgen.arkhipov@upol.cz

I fnori@riken.jp
}

\section{INTRODUCTION}

Exceptional points (EPs) are at the center of intense theoretical and experimental research in many different areas of physics, such as optics, condensed matter physics, plasmonics, and even electronics. Originally, 
EPs were studied in connection to parity-time $(\mathcal{P} \mathcal{T})$ symmetric non-conservative systems [1-3]. Since an EP corresponds to a non-diagonalizable operator, standard Hermitian Hamiltonians cannot display any EP. It is the nonunitary action of the environment on the system that induces the emergence of EPs. Such points have been studied by balancing attenuation, amplification, gain saturation, as well as various Hamiltonian coupling strengths of an open system (for experimental realizations, see e.g., Refs. [3-6]).

The interest in studying EPs, however, is not limited to $\mathcal{P} \mathcal{T}$-symmetric systems. Indeed, in proximity to other types of EPs, a system can display exotic phenomena, such as EP-induced lasing [3, 7, 8] or even new forms of photon blockade [9]. Beyond the purely phenomenological interest in studying these systems, EPs are considered for novel apparatus harnessing peculiar properties of mode coalescence at an EP $[10,11]$.

Many results obtained so far focused on non-Hermitian Hamiltonians (NHHs), i.e., systems in which losses, gain, and its saturation, decoherence, etc. are considered only as imaginary-valued fields. An EP of an NHH (which for brevity we refer to as a Hamiltonian EP or an HEP) refers to those $\mathrm{NHH}$ degeneracies where two (or more) eigenfrequencies coincide and the corresponding eigenstates coalesce. Arguably, one can categorize those systems as (semi)classical, since the effect of the environment has not been taken into account according to a full-quantum description.

Recently, the quest for true quantum EPs has attracted much attention [6, 9, 12-17]. An open quantum system interacting with its environment must include dissipative terms describing the progressive loss of energy, coherence, and information transfer to the environment [18-22]. For a weakly coupled Markovian environment, the (Gorini-Kossakowski-Sudarshan-Lindblad, or, for the sake of brevity) Lindblad master equation can efficiently capture the dynamics of the system. The Lindblad master equation consists of a Hermitian Hamiltonian part (i.e., the coherent evolution of the system) and the socalled Lindblad dissipators, which characterize the coupling with the environment [23]. These Lindblad dissipators can, in turn, be divided into two parts: the first one represents a coherent nonunitary dissipation of the system, similar to the imaginary-frequency fields of an $\mathrm{NHH}$; the second part describes quantum jumps, which result from the effect of a continuous measurement performed by the environment on the system [19, 21, 24, 25]. Quantum jumps induce both a random and instantaneous change of the stochastic wave function describing the system and a continuous non-Hermitian dynamics of the system [19, 21, 26-28]. Such instantaneous switching caused by quantum jumps is fundamental to obtain a theory of the system-environment interaction consistent with a probabilistic interpretation of quantummechanical measurements [20, 21, 27, 28]. Moreover, such quantum jumps have been experimentally observed in many setups [29-45].
To every Lindblad master equation corresponds a nonHermitian Liouvillian superoperator $\mathcal{L}$ which can display Liouvillian exceptional points (LEPs) [12, 23, 46-52]. LEPs are defined via degeneracies of Liouvillians (i.e., including the effects of quantum jumps) as introduced in Ref. [13] where their connection with HEPs has also been investigated (see also Refs. [9, 15]).

It has been noticed that an $\mathrm{NHH}$ naturally emerges when discussing quantum trajectories and postselection [6]. As mentioned above, quantum trajectories describe a system whose environment is continuously and perfectly probed. As mentioned above, quantum trajectories describe a system whose environment is continuously and perfectly probed. Even if quantum jumps cannot be avoided, if one postselects only those trajectories where no quantum jumps took place, the effective resulting dynamics is that of an NHH. For instance, in Ref. [6], such a postselection was used to explore the properties of the NHH of a superconducting three-level system.

From a Liouvillian point of view, postselecting the trajectories without quantum jumps corresponds to consider a Liouvillian without quantum jumps $\left(\mathcal{L}^{\prime}\right)$ [13]. Even if a perfect postselection of all quantum jumps is captured by $\mathcal{L}^{\prime}$, this ideal case may not be always realizable. For instance, one may be not able to postselect all the quantum jumps characterizing the system. Moreover, no real instrument can perfectly monitor the system, and therefore a perfect postselection is impossible. This motivates the question of how EPs and their associated effects depend on these protocols.

The main objective of this paper is to answer this question, addressing the relationship between HEPs, LEPs, and imperfectly postselected trajectories. For this, we introduce a hybrid Liouvillian $\mathcal{L}_{H}(q)$, a generalization of $\mathcal{L}$ and $\mathcal{L}^{\prime}$, capable of describing those (imperfect) processes. Roughly speaking, the quantum-jump parameter $q \in[0,1]$ describes how much one allows quantum jumps to affect the dynamics of a density matrix. This very formal definition allows to relate and compare HEPs with LEPs, and the corresponding evolutions between these two limits, i.e., the classical-to-quantum transition of EPs. By considering the protocol of quantum trajectories, we demonstrate that the hybrid Liouvillian $\mathcal{L}_{H}(q)$ has a very clear and specific physical meaning as the average over only a certain type of postselected trajectories. Indeed, $\mathcal{L}_{H}(q=0)$ represents a perfectly monitored system where we postselect only those trajectories where no quantum jump occurred (thus recovering an $\mathrm{NHH}$ ). On the contrary, $\mathcal{L}_{H}(q=1)$ describes the average of trajectories where no postselection has been applied, thus recovering a full Liouvillian description. Finally, we attribute the case $0<q<1$ to a postselected system in the presence of an imperfect measurement instrument.

This paper is organized as follows. In Sec. II, we recall the basic elements of an open-system description in terms of Lindblad operators, define LEPs and HEPs, recall the unraveling of the master equation in terms of quantum trajectories, and summarize postselection. In Sec. III, 


\begin{tabular}{c|c} 
Full Name & Abbreviation \\
\hline \hline Non-Hermitian Hamiltonian & NHH \\
\hline Liouvillian & $\mathcal{L}$ \\
\hline Liouvillian without quantum jumps & $\mathcal{L}^{\prime}$ \\
\hline Exceptional point & EP \\
\hline Hamiltonian exceptional point & HEP \\
\hline Liouvillian exceptional point & LEP \\
\hline Hybrid Liouvillian & $\mathcal{L}_{H}(q)$ \\
\hline Quantum jump parameter & $q$
\end{tabular}

Table I. List of abbreviation used in the main article, and corresponding full names.

we introduce the hybrid-Liouvillian formalism and provide two interpretations for it. In Secs. IV and V we discuss instructive examples of the classical-to-quantum transition of EPs. Finally, we discuss the implications of our results in the Conclusions. In the Appendix A we also provide the analytical expressions for the EPs in one of the studied examples of the hybrid Liouvillian.

In the main article, we will use all the previously introduced abbreviations. In Table I we concisely list them, to facilitate the reading of the article.

\section{LIOUVILLIANS AND QUANTUM TRAJECTORIES}

An open quantum system weakly interacting with a Markovian (i.e., memoryless) environment can be described using a Lindblad master equation:

$$
\partial_{t} \hat{\rho}(t)=-i[\hat{H}, \hat{\rho}(t)]+\sum_{\mu} \mathcal{D}\left[\hat{\Gamma}_{\mu}\right] \hat{\rho}(t)
$$

Here, $\hat{\rho}(t)$ represents the density matrix of the system, that is, the operator encoding all the information available to an external observer without any knowledge (or control) of how the environment microscopically and instantaneously acts on the system. The operator $\hat{H}$ is the Hamiltonian, describing the coherent evolution of the system, while $\hat{\Gamma}_{\mu}$ are quantum jump operators, describing the overall effect of the environment on the dynamics of the system. The jump operators act via the Lindblad dissipators as

$$
\mathcal{D}\left[\hat{\Gamma}_{\mu}\right] \hat{\rho}(t)=\hat{\Gamma}_{\mu} \hat{\rho}(t) \hat{\Gamma}_{\mu}^{\dagger}-\frac{1}{2}\left[\hat{\Gamma}_{\mu}^{\dagger} \hat{\Gamma}_{\mu} \hat{\rho}(t)+\hat{\rho}(t) \hat{\Gamma}_{\mu}^{\dagger} \hat{\Gamma}_{\mu}\right] .
$$

The effect of $\mathcal{D}\left[\hat{\Gamma}_{\mu}\right]$ on the density matrix $\hat{\rho}(t)$ can be split into two parts [21]: the continuous non-unitary dissipation terms, $\hat{\Gamma}_{\mu}^{\dagger} \hat{\Gamma}_{\mu} \hat{\rho}(t)+\hat{\rho}(t) \hat{\Gamma}_{\mu}^{\dagger} \hat{\Gamma}_{\mu}$, and the quantum jump terms $\hat{\Gamma}_{\mu} \hat{\rho}(t) \hat{\Gamma}_{\mu}^{\dagger}$. This dissipation describes the continuous and small loss of energy, information, and coherence of the system into the environment. The quantum jumps describe the abrupt changes of the state of the system due to dissipation, and can be thought of as the measurementlike action of a macroscopic environment on the state of the system [19, 21].

Equation (1) is linear in $\hat{\rho}(t)$ and, consequently, one can associate a superoperator to it - the so-called Liouvillian superoperator $\mathcal{L}$ [23]. This is a superoperator in the sense that it acts on an operator (the density matrix) to produce an operator analogous to the way in which an operator acts on a vector to produce a new vector [27]. Using the Liouvillian $\mathcal{L}$, we have

$$
\partial_{t} \hat{\rho}(t)=\mathcal{L} \hat{\rho}(t)
$$

Hereafter, we assume that $\mathcal{L}$ is time independent.

\section{A. Liouvillian spectrum, Hamiltonian and Liouvillian EPs}

The spectrum of the Liouvillian $\mathcal{L}$ is found according to the formula

$$
\mathcal{L} \hat{\rho}_{i}=\lambda_{i} \hat{\rho}_{i}
$$

where $\lambda_{i}$ and $\hat{\rho}_{i}$ are the eigenvalues and eigenmatrices of the Liouvillian, respectively. For convenience, we sort the eigenvalues in such a way that $\left|\mathbb{R e}\left[\lambda_{0}\right]\right|<\left|\mathbb{R e}\left[\lambda_{1}\right]\right|<$ $\ldots<\left|\mathbb{R e}\left[\lambda_{n}\right]\right|$.

The models which we consider are finite dimensional and time independent. In this regard, one is guaranteed that there always exists a zero eigenvalue of the Liouvillian. This represents the fact that there exists at least one steady state $\hat{\rho}_{\mathrm{ss}}$ towards which the system evolves. Even if the steadystate density matrix associated with the zero is important in many aspects of open quantum systems, in this case it is not particularly interesting since it cannot display any exceptional point (see the discussion in Ref. [13]).

For a Liouvillian with a unique steady state, the eigenmatrix $\hat{\rho}_{0}$, associated with $\lambda_{0}=0$, defines the steadystate density matrix $\hat{\rho}_{\mathrm{ss}} \propto \hat{\rho}_{0}$ of the system. The eigenmatrices $\hat{\rho}_{i}$, with $i>0$, describe the transient dynamics towards the steady state. For a more detailed discussion of the properties of the Liouvillian spectrum, see Refs. [13, 23, 46-48].

In this formalism, LEPs describe the coalescence of two eigenmatrices of the Liouvillian, for some appropriate choice of parameters. At an EP, the Liouvillian is defective and cannot be diagonalized. For a LEP of order 2 , the eigenvalue $\lambda_{j}$ admits only one eigenmatrix $\hat{\rho}_{j}$. However, one can introduce a generalized eigenmatrix $\hat{\tilde{\rho}}_{j}$, which is defined via the Jordan chain:

$$
\mathcal{L} \hat{\tilde{\rho}}_{j}=\lambda_{j} \hat{\tilde{\rho}}_{j}+\hat{\rho}_{j} .
$$

This generalized eigenmatrix completes the basis of the other eigenmatrices $\hat{\rho}_{i}$, i.e., any operator can be written as a linear combination of the $\hat{\rho}_{i}$ and $\hat{\tilde{\rho}}_{j}$. In this basis, the Liouvillian attains its Jordan canonical form. 
One of the central results proved in Ref. [13] is that LEPs should be understood as purely dynamical phenomena since in this Lindblad ME formalism, LEPs can emerge only for the "excitations" $\hat{\rho}_{i}(i>0)$ above the steady state $\hat{\rho}_{0}$.

Given a Lindblad master equation as in Eq. (1), we can introduce the corresponding effective $\mathrm{NHH}$ of the form

$$
\hat{H}_{\mathrm{eff}}=\hat{H}-i \sum_{\mu} \frac{\hat{\Gamma}_{\mu}^{\dagger} \hat{\Gamma}_{\mu}}{2} .
$$

Note that $\hat{H}_{\text {eff }}$ is a NHH since $\hat{H}_{\text {eff }}^{\dagger} \neq \hat{H}_{\text {eff }}$. The equation of motion for a generic density matrix $\hat{\rho}(t)$, thus, becomes

$$
\frac{\partial \hat{\rho}(t)}{\partial t}=\mathcal{L} \hat{\rho}(t)=-i\left[\hat{H}_{\mathrm{eff}} \hat{\rho}(t)-\hat{\rho}(t) \hat{H}_{\mathrm{eff}}^{\dagger}\right]+\sum_{\mu} \hat{\Gamma}_{\mu} \hat{\rho}(t) \hat{\Gamma}_{\mu}^{\dagger} .
$$

If one assumes that the effect of the jump operators $\sum_{\mu} \hat{\Gamma}_{\mu} \hat{\rho}(t) \hat{\Gamma}_{\mu}^{\dagger}$ is always zero during this evolution, the evolution of the system is provided by $\hat{H}_{\text {eff }}$. Such nonHermitian operators may support EPs in their spectra, which we refer to here as NHH Hamiltonian EPs (HEPs) in contrast to LEPs.

\section{B. Quantum trajectories}

From a theoretical point of view, there are two very different physical interpretations which can be associated with the Lindblad master equation. The first interpretation is to consider that a true action of the environment on the system is impossible to be known exactly, so that the dissipators $\mathcal{D}\left[\hat{\Gamma}_{\mu}\right]$ describe the average effect of the environment. In this sense, the density matrix $\hat{\rho}(t)$ is a statistical mixture since one does not know the details of the system-environment interaction.

On the contrary, if we were to know perfectly the action of the system on the environment, then we could model it as a series of perfect measurement instruments $[18,19,21]$. In this description, the action of the dissipators $\mathcal{D}\left[\hat{\Gamma}_{\mu}\right]$ is to induce random changes in the system (associated with the detection of one of the operators $\hat{\Gamma}_{\mu}$ ). Once an average over several realizations of the same protocol is considered, the randomness associated with the dissipator action introduces a statistical mixture of pure states, resulting in the density matrix $\hat{\rho}(t)$.

Both approaches lead to the same average results [19, 21]; i.e., the average descriptions of the system evolution are equivalent. However, while according to the first interpretation it is conceptually difficult to consider the state of a quantum system during a single experimental realization, the second approach allows to describe an idealized evolution of the system whose environment is continuously and perfectly probed (or monitored). Such an equation of motion is called a quantum trajectory (for a more detailed discussion, see, e.g., Refs. [26-28, 53, 54]). In this formalism, the state of the system along a trajectory is described by a wave function $|\psi(t)\rangle$, which evolves stochastically, and the results of the Lindblad master equation are recovered by averaging over many trajectories.

Theoretically, the simplest measurement instrument continuously monitoring the environment is the one which produces only two outcomes: one if the desired state is detected, zero otherwise. We can imagine that, for each dissipator, there is an instrument measuring if a quantum jump takes place continuously, perfectly, and instantaneously. By counting the number of quantum jumps which are taking place of each type, we can reconstruct the state of a given system [21]. Using this trajectory counting apparatus for an infinitesimal time $\mathrm{d} t$, the evolution of the wave function can be described by:

$$
\begin{aligned}
\mathrm{d}|\psi(t)\rangle= & {\left[\sum_{\mu} \mathrm{d} N_{\mu}(t)\left(\frac{\hat{\Gamma}_{\mu}}{\sqrt{\left\langle\hat{\Gamma}_{\mu}^{\dagger} \hat{\Gamma}_{\mu}\right\rangle}}-\mathbb{1}\right)\right.} \\
& \left.+\mathrm{d} t\left(-i \hat{H}_{\mathrm{eff}}+\sum_{\mu} \frac{\left\langle\hat{\Gamma}_{\mu}^{\dagger} \hat{\Gamma}_{\mu}\right\rangle}{2}\right)\right]|\psi(t)\rangle,
\end{aligned}
$$

where the effective Hamiltonian is that introduced in Eq. (6).

Note that $\mathrm{d}|\psi(t)\rangle$ is a differential describing either the abrupt evolution with a stochastic quantum jump [the term proportional to $\left.\mathrm{d} N_{\mu}(t)\right]$ or the smooth nonunitary evolution dictated by $\hat{H}_{\text {eff }}$. The expectation values of the jump operators, instead, ensure that the wavefunction $\mathrm{d}|\psi(t)\rangle$ remains well-normalized along the dynamics. For a more detailed discussion, see Eq. (4.17) of Ref. [21].

The stochastic "counting" parameters $N_{\mu}(t)$ contain the information about the total number of jumps which took place along the dynamics from the initial time $t=0$ to time $t$. Hence, $\mathrm{d} N_{\mu}(t)=N_{\mu}(t+\mathrm{d} t)-N_{\mu}(t)$ is the dichotomic stochastic variable representing the detection outcome at time $t$. Specifically, $\mathrm{d} N_{\mu}(t)=0\left[\mathrm{~d} N_{\mu}(t)=1\right]$ if no (one) quantum jump $\hat{\Gamma}_{\mu}$ took place. Hence, one cannot simply take the derivative of $\mathrm{d} N(t)$ with respect to $\mathrm{d} t$. One can, however, define the probability that a quantum jump occurs during a time $\mathrm{d} t$ as

$$
p\left[\mathrm{~d} N_{\mu}(t)=1\right]=\left\langle\hat{\Gamma}_{\mu}^{\dagger} \hat{\Gamma}_{\mu}\right\rangle \mathrm{d} t .
$$

The NHH can thus be interpreted as the operator determining the dynamics between two successive quantum jumps. Furthermore, the terms $\left\langle\hat{\Gamma}_{\mu}^{\dagger} \hat{\Gamma}_{\mu}\right\rangle$ in Eq. (8) act as normalization constants necessary to ensure that $\langle\Psi(t) \mid \Psi(t)\rangle=1$. Equivalently, we can think that the system evolves under the action of $\hat{H}_{\text {eff }}$ and we have to renormalize the wave function at each time step. The above interpretation allows also for simple efficient Monte Carlo simulation of the ensuing dynamics [19, 21, 26, 55, 56].

Finally, we note that the trajectory-counting-based monitoring of the operators $\hat{\Gamma}_{\mu}$ is not the only possible unraveling of the master equation. Indeed, there exist other possible choices of jump operators which result in the same master equation once the average over many 
quantum trajectories is taken [19, 21]. Different unraveling can result in extremely different dynamics at a single trajectory level $[19,57,58]$. In this sense, the use of a Lindblad master equation allows to capture those properties which do not depend on the details of the systemenvironment exchange.

\section{Postselection of quantum trajectories}

Suppose now that we observe a quantum trajectory where, at time $t, N_{\mu}(t)=0$ for all $\mu$. We conclude that the system has evolved under the genuine action of the NHH $\hat{H}_{\text {eff }}$ in Eq. (8). In this regard, by postselecting the trajectory with no quantum jumps (i.e., discarding all those which do present some quantum jumps) one can obtain an $\mathrm{NHH}$ also in the quantum limit, that is, when normally quantum jumps would play a fundamental role in correctly describing the physics of the system. As has been shown in Ref. [6], this procedure allows to study the emergence of HEPs also in quantum systems.

There are, however, some necessary remarks concerning this postselection procedure. First, we notice that, in this way, we cannot experimentally connect manyparticle semiclassical EPs to the fully quantum ones. Indeed, in the semiclassical limit of a many-particle system, many quantum jumps must happen, and the probability to observe a trajectory without quantum jumps rapidly tends to zero.

Indeed, postselecting in the semiclassical limit would be equivalent to avoiding environment-induced superselection (einselections), collapsing the "quantum" wave function into a classical state [59]. For example, in an optical cavity with jump operator $\hat{a}$ ( $\hat{a}$ being the bosonic destruction operator), the number of jumps per unit of time $\mathrm{d} t$ is roughly given by $\mathrm{d} t \cdot\left\langle\Psi(t)\left|\hat{a}^{\dagger} \hat{a}\right| \Psi(t)\right\rangle$. In a manyparticle system, this number is extremely high, making it almost impossible to observe a trajectory without quantum jumps.

Moreover, to truly observe a HEP it is necessary to have a perfect measurement instrument which collects all the quantum jumps and that never allows a quantum jump to go undetected. Hence, in principle, true postselection is impossible, which leads to two questions:

(i) How can we relate the results of NHHs and Liouvillians in a more formal way?

(ii) How can we describe the consequences of imperfect monitoring, i.e., finite efficiency detectors in quantum trajectories?

\section{HYBRID-LIOUVILLIAN FORMALISM AND ITS CONNECTION TO POSTSELECTION}

To answer both questions raised in the previous section, we introduce a hybrid-Liouvillian formalism; i.e., we introduce a modified Liouvillian superoperator. To better understand this hybrid Liouvillian, here we focus on Eq. (3) which in the case where there is just one quantum jump becomes

$$
\mathcal{L}=-i[\hat{H}, \cdot]+\hat{\Gamma} \cdot \hat{\Gamma}^{\dagger}-\frac{\hat{\Gamma}^{\dagger} \hat{\Gamma} \cdot+\cdot \hat{\Gamma}^{\dagger} \hat{\Gamma}}{2}
$$

where - is a placeholder to indicate where an operator should be applied. As already mentioned, ignoring the effect of quantum jumps $\hat{\Gamma} \cdot \hat{\Gamma}^{\dagger}$, one obtains a non-Hermitian Hamiltonian evolution which can be recast in superoperator form as

$$
\mathcal{L}^{\prime}=-i\left(\hat{H}-i \frac{\hat{\Gamma}^{\dagger} \hat{\Gamma}}{2}\right) \cdot+i \cdot\left(\hat{H}-i \frac{\hat{\Gamma}^{\dagger} \hat{\Gamma}}{2}\right)^{\dagger}=-i \hat{H}_{\mathrm{eff}} \cdot+i \cdot \hat{H}_{\mathrm{eff}}^{\dagger}
$$

The superoperator $\mathcal{L}^{\prime}$ is the Liouvillian without quantum jumps, and its spectrum is fully determined by that of $\hat{H}_{\text {eff }}$ [13]. This equation is not trace preserving. This problem is solved as in the case of evolution with $\hat{H}_{\text {eff }}$ in a quantum trajectory, where the density matrix can be renormalized at each time step to ensure that $\operatorname{Tr}[\hat{\rho}(t)]=1$. Now, by not completely ignoring the effects of quantum jumps, one can formally introduce a hybrid Liouvillian of the form:

$$
\begin{aligned}
\mathcal{L}_{\mathrm{H}}(q) & =-i[\hat{H}, \cdot]+q \hat{\Gamma} \cdot \hat{\Gamma}^{\dagger}-\frac{\hat{\Gamma}^{\dagger} \hat{\Gamma} \cdot+\cdot \hat{\Gamma}^{\dagger} \hat{\Gamma}}{2} \\
& =-i[\hat{H}, \cdot]+\mathcal{D}[\sqrt{q} \hat{\Gamma}]-(1-q) \frac{\hat{\Gamma}^{\dagger} \hat{\Gamma} \cdot+\cdot \hat{\Gamma}^{\dagger} \hat{\Gamma}}{2}
\end{aligned}
$$

The hybrid Liouvillian $\mathcal{L}_{\mathrm{H}}(q)$ has a clear mathematical significance, viz., it is the weighted average of $\mathcal{L}$ and $\mathcal{L}^{\prime}$. Clearly it interpolates between $\mathrm{NHH}$ evolution $(q=0)$ to a completely Liouvillian one $(q=1)$ and the transition of EPs between these two limits can be traced by tuning the quantum jump parameter $q$. The physical meaning of this hybrid operator is explained below.

\section{A. Interpretation of the hybrid Liouvillian in terms of post-selected trajectories}

The Liouvillian in Eq. (10) can be conveniently recast as:

$$
\begin{aligned}
\mathcal{L} & =-i[\hat{H}, \cdot]+\mathcal{D}[\hat{\Gamma}]=-i[\hat{H}, \cdot]+q \mathcal{D}[\hat{\Gamma}]+(1-q) \mathcal{D}[\hat{\Gamma}] \\
& =-i q[\hat{H}, \cdot]+\mathcal{D}[\sqrt{q} \hat{\Gamma}]-i(1-q)[\hat{H}, \cdot]+\mathcal{D}[\sqrt{1-q} \hat{\Gamma}]
\end{aligned}
$$

From a quantum trajectory point of view, Eq. (13) means that instead of having only one measuring instrument collecting the jumps of the operator $\hat{\Gamma}$, we have two different 

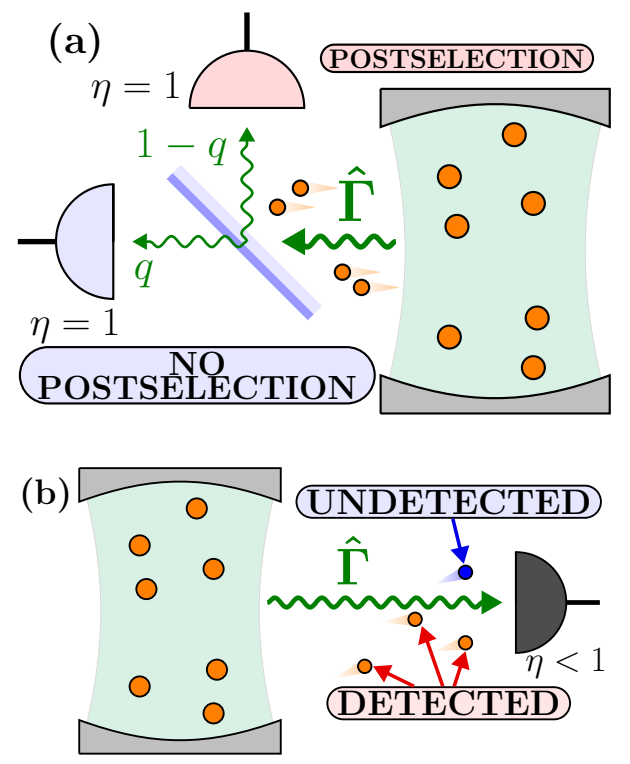

Figure 1. Pictorial representation of the physical systems for which evolution is described by the hybrid Liouvillian $\mathcal{L}_{H}(q)$ depending on the quantum jump parameter $q$. To clarify the ideas, we sketch an optical cavity with one-photon loss as dissipator (the jump operator $\hat{\Gamma}$ ). (a) A system with two perfect photodetectors (efficiency $\eta=1$ ) simultaneously measuring the same jump operator $\hat{\Gamma}$ for a photon leaking from the cavity and passing through a beam splitter (BS) with the transmission (reflection) probability $q(1-q)$. Thus, the quantum jump parameter $q$ corresponds to the probability of measuring a leaking photon by the left photodetector. According to this setup, we postselect only those trajectories which read $N^{(2)}(t)=0$; that is, no quantum jumps were detected in the photodetector (detecting photons with probability $1-q$, the upper one in the drawing). (b) A finite-efficiency photodetector $(\eta<1)$. While a certain fraction of the photons will be detected (the orange ones), some of them will not (the blue one). We then perform a postselection requiring that no quantum jumps were detected. Since not all the photons which escaped the system where detected, we may average also over some quantum trajectories where a photon escaped the system. This hybrid Liouvillian describing this system is $\mathcal{L}_{H}(q=1-\eta)$.

detectors [c.f. Fig. 1 (a)], so that

$$
\begin{aligned}
\mathrm{d}|\psi(t)\rangle=\{ & {\left[\left(\mathrm{d} N^{(1)}(t)+\mathrm{d} N^{(2)}(t)\right)\left(\frac{\hat{\Gamma}}{\sqrt{\left\langle\hat{\Gamma}^{\dagger} \hat{\Gamma}\right\rangle}}-\mathbb{1}\right)\right] } \\
& \left.-i \mathrm{~d} t\left(\hat{H}_{\mathrm{eff}}+\frac{\left\langle\hat{\Gamma}^{\dagger} \hat{\Gamma}\right\rangle}{2}\right)\right\}|\psi(t)\rangle,
\end{aligned}
$$

where $\hat{H}_{\text {eff }}$ is

$$
\begin{aligned}
\hat{H}_{\mathrm{eff}} & =\hat{H}_{\mathrm{eff}}^{(1)}+\hat{H}_{\mathrm{eff}}^{(2)}, \\
\hat{H}_{\mathrm{eff}}^{(1)} & =\hat{H}-i q \frac{\hat{\Gamma}^{\dagger} \hat{\Gamma}}{2}, \\
\hat{H}_{\mathrm{eff}}^{(2)} & =\hat{H}-i(1-q) \frac{\hat{\Gamma}^{\dagger} \hat{\Gamma}}{2} .
\end{aligned}
$$

The total probability of a jump in an infinitesimal amount of time is given by

$$
p\left[\mathrm{~d} N^{(1)}(t)+\mathrm{d} N^{(2)}(t)=1\right]=\left\langle\hat{\Gamma}^{\dagger} \hat{\Gamma}\right\rangle \mathrm{d} t .
$$

and

$$
\begin{aligned}
& p\left[\mathrm{~d} N^{(1)}(t)=1\right]=q\left\langle\hat{\Gamma}^{\dagger} \hat{\Gamma}\right\rangle \mathrm{d} t, \\
& p\left[\mathrm{~d} N^{(2)}(t)=1\right]=(1-q)\left\langle\hat{\Gamma}^{\dagger} \hat{\Gamma}\right\rangle \mathrm{d} t .
\end{aligned}
$$

That is, Eqs. (14), (15), and (17) correspond to assuming that a fraction $q$ of detections will happen in the first detector, while all the other detections happen in the second one. The average total number of detections in an experiment, however, must be identical to a setup where only one detector is present [cf. Eq. (16)].

Let us assume that we postselect the results of the second detector; i.e., we choose only those trajectories where no quantum jump took place for the second detector and $N^{(2)}(t)=0$. Detector 1 will exactly produce the Lindblad master equation with dissipator $\mathcal{D}[\sqrt{q} \hat{\Gamma}]$, while the second detector will produce a time evolution via its non-Hermitian Hamiltonian $\hat{H}_{\text {eff }}^{(2)}$. Hence the Liouvillian in Eq. (13) becomes

$$
-i[\hat{H}, \cdot]+\mathcal{D}[\sqrt{q} \hat{\Gamma}]-(1-q) \frac{\hat{\Gamma}^{\dagger} \hat{\Gamma} \cdot+\cdot \hat{\Gamma}^{\dagger} \hat{\Gamma}}{2} \equiv \mathcal{L}_{H}(q)
$$

We have therefore proved that $\mathcal{L}_{H}(q)$ describes the evolution of the state monitored by two perfect instruments, one of which is post-selected. Hence, $\mathcal{L}_{H}(q)$ is a physically legitimate quantum map. Note that one must, however, renormalize the density matrix to ensure that its trace is 1 .

\section{B. Postselected quantum trajectory and inefficient detectors}

We can also assign a different, experimentally relevant meaning to Eq. (14). Let us consider a finite-efficiency detector, such that, with probability $\eta$, a quantum jump happens but the detector does not report it happening. The Lindblad master equation of this system is (see, e.g., page 190 of Ref. [21]):

$$
\mathcal{L}(\eta)=-i[\hat{H}, \cdot]+\mathcal{D}[\hat{\Gamma}]=-i[\hat{H}, \cdot]+\eta \mathcal{D}[\hat{\Gamma}]+(1-\eta) \mathcal{D}[\hat{\Gamma}] .
$$

Again, we can model such a system as one in which we have two perfect detectors which continuously monitor 
the system and collect all the quantum jumps which take place. Even if, theoretically, the effects of these two detectors on a single quantum trajectory are identical to the presence of a single detector, the description is extremely different once we try to postselect the results. Indeed, one of the two detectors does not pass any information to an observer, which cannot know if a quantum jump took place. The description of such a system is, therefore, exactly captured by the hybrid Liouvillian $\mathcal{L}_{H}(q)$, where now $q=1-\eta$ depends on the detector efficiency.

\section{The $q>1$ case}

As we previously discussed, we can produce an $\mathrm{NHH}$ by considering $q=0$. In this case, the dynamics of the system is completely determined by $\hat{H}_{\text {eff }}$. Thus, studying $\mathcal{L}_{H}(0)$ we can infer if the effect of the $\mathrm{NHH}$ is to create or destroy an EP.

However, we cannot know in this formalism what is the effect of only the quantum jumps on the system. To do that, one should consider the $q \rightarrow \infty$ limit, where the NHH can never act on the system. From the previous discussion it is clear that, to ensure a correct interpretation of the hybrid-Liouvillian in terms of post-selected trajectories, we need $q \in[0,1]$. Therefore, $\mathcal{L}_{H}(q>1)$ cannot be obtained by simply considering a post-selection procedure.

Even if we cannot provide a clear physical interpretation to $\mathcal{L}_{H}(q>1)$, we can still study what happens to the spectrum of $\mathcal{L}_{H}(q \gg 1)$ mathematically. In this limit, the overall evolution of the system is given by the quantum jump operator, and the fate of the EPs of $\mathcal{L}_{H}(q \rightarrow \infty)$ tells us if quantum jumps either favor or are detrimental for the emergence of EPs.

\section{EXAMPLE 1: A MODEL WITH A LEP WITHOUT HEPS}

In this Section, we consider a simplified version of the model exhibiting LEPs but not HEPs given in Ref. [13]. We consider a spin-1/2, with Hamiltonian

$$
\hat{H}=\frac{\omega}{2} \hat{\sigma}_{z},
$$

which evolves under the action of the decay channel $\hat{\sigma}_{x}$, i.e.,

$$
\mathcal{L} \hat{\rho}(t)=-i[\hat{H}, \hat{\rho}(t)]+\frac{\gamma_{x}}{2} \mathcal{D}\left[\hat{\sigma}_{x}\right]
$$

where $\hat{\sigma}_{x, z}$ are the Pauli matrices. Since this master equation is invariant under the exchange $\hat{\sigma}_{-} \rightarrow-\hat{\sigma}_{-}$, this model explicitly presents a $\mathcal{Z}_{2}$ symmetry [46, 47].

As discussed in Ref. [13], the NHH structure is trivial and cannot present any EPs, since

$$
\hat{H}_{\mathrm{eff}}=\frac{\omega}{2} \sigma_{z}-i \gamma_{x} \mathbb{1}
$$

is already diagonal, and its two eigenvalues are always different. The Liouvillian, instead, can present several interesting properties. We have the eigenvalues

$$
\left\{\begin{aligned}
\lambda_{0} & =0, \\
\lambda_{1,2} & =-\gamma_{x} \pm \Omega, \\
\lambda_{3} & =-2 \gamma_{x},
\end{aligned}\right.
$$

and the corresponding eigenmatrices

$$
\left\{\begin{aligned}
\hat{\rho}_{0} & \propto \hat{\rho}_{\mathrm{ss}}=\frac{1}{2 \gamma_{x}}\left(\begin{array}{cc}
\gamma_{x} & 0 \\
0 & \gamma_{x}
\end{array}\right), \\
\hat{\rho}_{1,2} & \propto\left(\begin{array}{cc}
0 & -i \omega \pm \Omega \\
\gamma_{x} & 0
\end{array}\right), \\
\hat{\rho}_{3} & \propto\left(\begin{array}{cc}
-1 & 0 \\
0 & 1
\end{array}\right)
\end{aligned}\right.
$$

where $\Omega=\sqrt{\gamma_{x}^{2}-\omega^{2}}$ and $\hat{\rho}_{\text {ss }}$ is the steady-state density matrix. Therefore, this Liouvillian exhibits an EP for $\gamma_{x}^{\mathrm{EP}}=\omega$. For the model under consideration, we obtain a family of solutions for the generalized eigenmatrix $\hat{\tilde{\rho}}_{1}$ depending on one parameter $a$ [c.f. Eq. (5)]:

$$
\hat{\tilde{\rho}}_{1}=\left(\begin{array}{cc}
0 & a \\
i a-i & 0
\end{array}\right) \text {. }
$$

\section{A. Equivalence between postselection trajectories and hybrid Liouvillian}

Here, we show the equivalence between postselection and the hybrid Liouvillian $\mathcal{L}_{H}(q)$ stemming from Eq. (21):

$$
\mathcal{L} \hat{\rho}(t)=-i[\hat{H}, \hat{\rho}(t)]+q \frac{\gamma_{x}}{2} \mathcal{D}\left[\hat{\sigma}_{x}\right]-\frac{(1-q) \gamma_{x}}{2} \mathbb{1} \cdot
$$

\section{Two-detector post-selected trajectories}

In Fig. 2, we study the time evolution of a qubit both using $\mathcal{L}_{H}(q)$ and post-selected trajectories for the twodetector model [see also the sketch in Fig. 1 (a)]. The markers represent the results obtained by averaging over 5000 single quantum trajectories, from which we postselected only the evolutions activating a chosen detector. The error bars are associated with the statistical error due to the finite number of trajectories simulated. The curves, instead, have been obtained by evolving the initial state via $\mathcal{L}_{H}(q)$.

The algorithm to simulate this protocol for a number $N_{\text {traj }}$ of trajectories is the following:

Step 1: Simulate a quantum trajectory with two possible jump operators:

$$
\hat{\Gamma}_{1}=\sqrt{q \gamma_{x} / 2} \hat{\sigma}_{x}, \quad \text { and } \quad \hat{\Gamma}_{2}=\sqrt{(1-q) \gamma_{x} / 2} \hat{\sigma}_{x}
$$




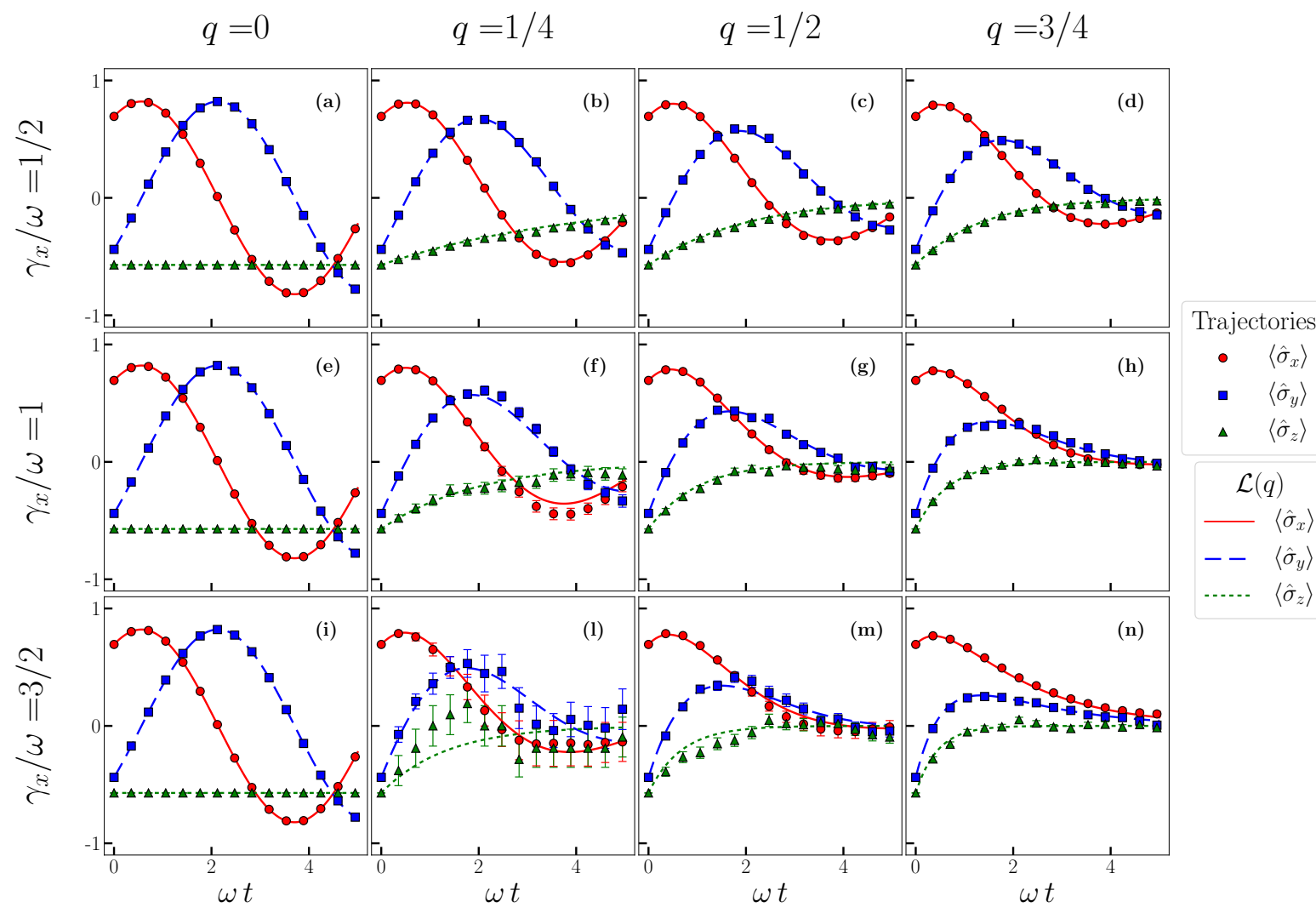

Figure 2. Two-detector post-selected trajectories [c.f. Fig. 1 (a)] vs evolution using $\mathcal{L}_{H}(q)$ for the two-level system in Eq. (26): as a function of time are plotted the expectation values of $\hat{\sigma}_{x}$ (red curves and markers), $\hat{\sigma}_{y}$ (blue curves and markers), and $\hat{\sigma}_{z}$ (green curves and markers). The markers represent the results obtained by the postselection of the trajectories, while the curves represent the results obtained via the hybrid Liouvillian: [(a), (e), (i)] $q=0,[(\mathrm{~b}),(\mathrm{f}),(\mathrm{l})] q=1 / 4 ;[(\mathrm{c}),(\mathrm{g}),(\mathrm{m})] q=1 / 2$, and $[(\mathrm{d}),(\mathrm{h}),(\mathrm{n})] q=3 / 4 ;(\mathrm{a}-\mathrm{d}) \gamma_{x}=\omega / 2,(\mathrm{e}-\mathrm{h}) \gamma_{x}=\omega$, and (i-n) $\gamma_{x}=3 \omega / 2$. The initial state is $|\psi\rangle=\cos (\theta / 2)|\uparrow\rangle+\sin (\theta / 2) e^{i \phi}|\downarrow\rangle$, for $\theta=\sqrt{3} \pi / 2$ and $\phi=\sqrt{3} \pi$. The data have been obtained by averaging over 5000 trajectories per parameter set (see the details about the algorithm in the main text). The error bars $\Delta\left\langle\hat{\sigma}_{i}\right\rangle$ for a generic operator $\hat{\sigma}_{i}$ have been obtained by computing the standard error of the mean $\Delta\left\langle\hat{\sigma}_{i}\right\rangle=\sqrt{\left\langle\hat{\sigma}_{i}-\left\langle\hat{\sigma}_{i}\right\rangle\right\rangle^{2}} / \sqrt{N-1}$, with $N$ representing the number of trajectories on which the average was taken.
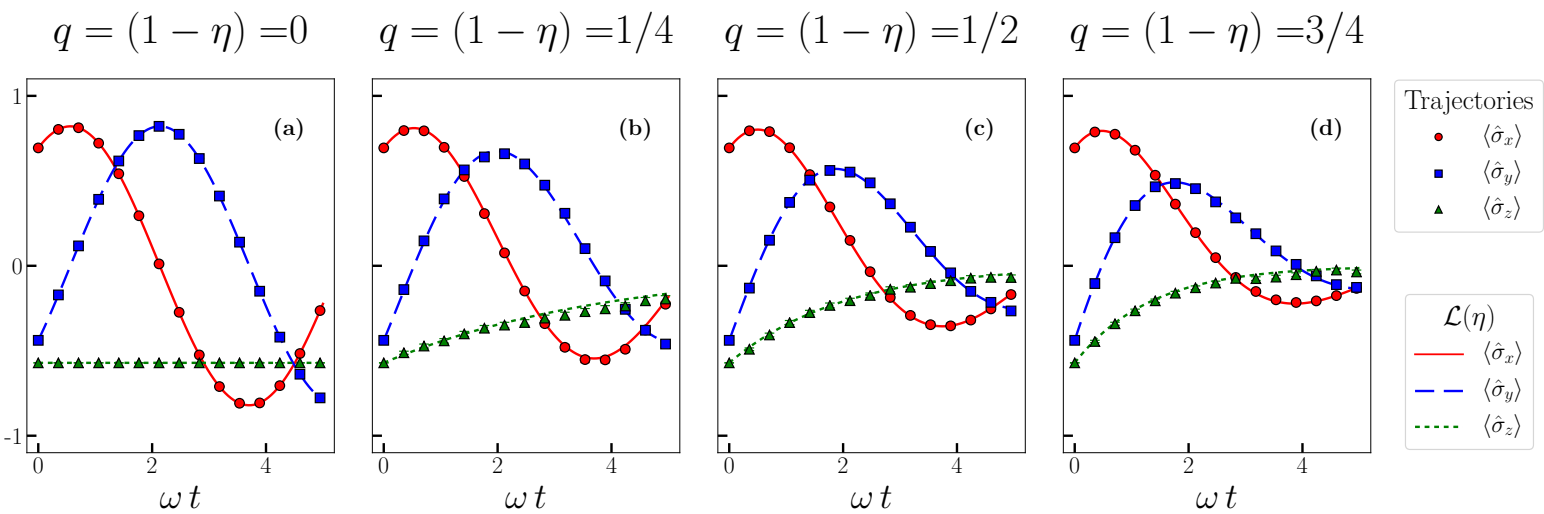

Figure 3. Inefficient detector [c.f. Fig. 1 (b)] vs evolution using $\mathcal{L}_{H}(q)=\mathcal{L}_{H}(1-\eta)$ for the two-level system in Eq. (26) for $\gamma_{x}=\omega / 2$. As a function of time, we plot the expectation values of $\hat{\sigma}_{x}$ (red lines and markers), $\hat{\sigma}_{y}$ (blue lines and markers), and $\hat{\sigma}_{z}$ (green lines and markers). The markers represent the results obtained by the postselection of the trajectories, while the curves represent the results obtained via the hybrid Liouvillian: (a) $q=0(\eta=1)$, (b) $q=1 / 4(\eta=3 / 4),(\mathrm{c}) q=1 / 2(\eta=1 / 2)$, and (d) $q=3 / 4(\eta=1 / 4)$. The data have been obtained by averaging over 5000 trajectories per parameter set (see the details about the algorithm in the main text) and the initial state is the same as in Fig. 2. The error bars have been calculated as in Fig. 2. 


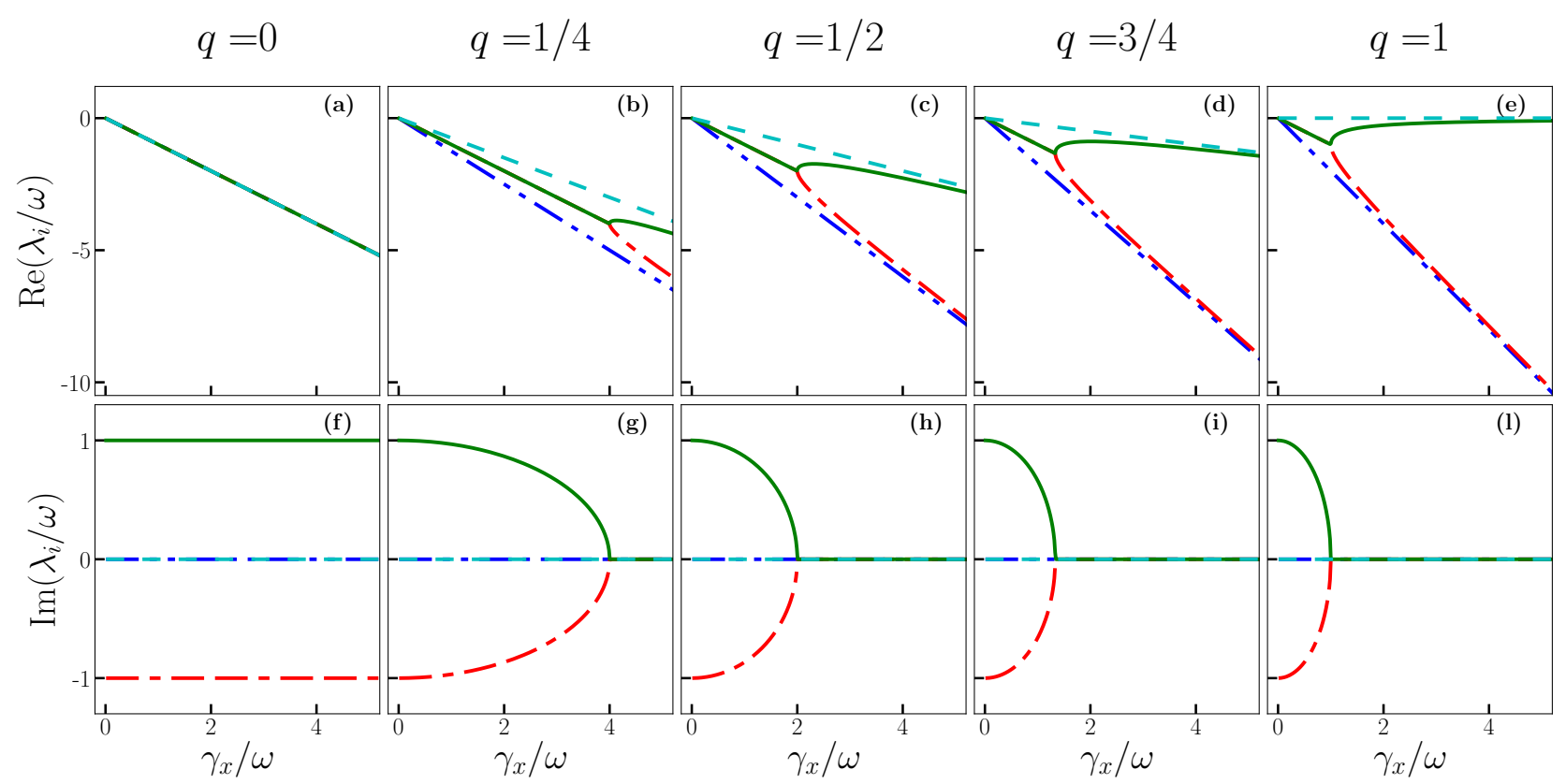

Figure 4. Spectrum of the hybrid-Liouvillian superoperator in Eq. (26) as a function of the rescaled dissipation rate $\gamma_{x} / \omega$. (a-e) The real part of the eigenvalues $\lambda_{i}$, and panels (f-l) the imaginary part. [(a), (f)] $q=0$ (the NHH case), [(b), (g)] $q=1 / 4,[(\mathrm{c})$, (h)] $q=1 / 2,[(\mathrm{~d}),(\mathrm{i})] q=3 / 4$, and [(e), (l)] $q=1$ [i.e., diagonalization of the Liouvillian $\mathcal{L}$ in Eq. (21)].

Step 2: Once the simulation of one trajectory is completed, check the total number of jumps which took place in $\hat{\Gamma}_{2}$, i.e., $N^{(2)}(t)$ : if $N^{(2)}(t)=0$, save the trajectory, otherwise reject it;

Step 3: Once $N_{\text {traj }}$ trajectories have been simulated, average only on the correct one.

Figures. 2(a), 2(e), and 2(i) (left column) represent the results for $q=0$, that is, no detection occurred, for $\gamma_{x}=\omega / 2, \gamma_{x}=\omega$, and $\gamma_{x}=3 \omega / 2$, respectively. Correctly, the evolution is identical for the three different values of $\gamma_{x}$, as it stems from the NHH. If we consider now panels 2(b)-2(d), which represent $\gamma_{x}=\omega / 2$, we observe a perfect agreement between the trajectories' behavior and that of $\mathcal{L}_{H}(q)$, demonstrating the validity of the previous discussion. Note also that changing $q$ produces a sizable effect on the system. If we increase the value of $\gamma_{x}$ [panels (f-h), $\left.\gamma_{x}=\omega\right]$ there are some deviation from the results of the hybrid Liouvillian. This noise is due to the fact that, by increasing $\gamma_{x}$, the mean number of quantum jumps increases and, therefore, fewer and fewer trajectories which can be postselected. This effect becomes evident in Figs. 2(i)-2(n), where $\gamma_{x}=3 \omega / 2$. In particular, in Fig. 2(1), out of the 5000 trajectories which we simulated, only 15 could be averaged. Moreover, the results in Fig. 2(n) are far less noisy than those in Fig. 2(l): a higher $q$ means a smaller rejection rate, and therefore we can average over a much higher number of trajectories.

\section{Imperfect detection}

Similarly to the previous case, in Fig. (3) we consider now an imperfect detector, similar to the one sketched in Fig. 1(b). The detector efficiency $\eta$ represents the probability that when a quantum jump happens it is detected. Thus, $\eta=1$ is a perfect detector, and all the quantum jumps are detected. We simulate 5000 trajectories and we average only on those which, according to our imperfect photodetector, had zero quantum jumps.

The algorithm simulating this imperfect detection for a number $N_{\text {traj }}$ of trajectories is the following:

Step 1: Simulate a quantum trajectory with one jump operator:

$$
\hat{\Gamma}=\sqrt{\gamma_{x} / 2} \hat{\sigma_{x}}
$$

Step 2: Once the simulation of one trajectory is performed, we store those trajectories where all the quantum jumps which took place have not been detected, or no jump took place. To do that, we count the number $N_{j}$ of quantum jumps. We extract an array of $N_{j}$ random numbers $\left\{n_{j} \in[0,1]\right\}$, representing the aleatory nature of the imperfect detector in non-detecting the quantum jump. If, for all $j, n_{j}>\eta$ (or, equivalently, $n_{j}>1-q$ ) we save the trajectory. Otherwise reject it.

Step 3: Once $N_{\text {traj }}$ trajectories have been simulated, average only on the correct ones. 
Again, in Fig. (3) we see an excellent agreement between the postselected averaged quantum-trajectory (markers) and the evolution via operator $\mathcal{L}_{H}(q)=\mathcal{L}_{H}(1-$ $\eta)$ (curves). We have therefore demonstrated the validity of our proposed protocol and its physical meaning also in describing the physics of an imperfect detector.

\section{B. Transition of NHH to $\mathcal{L}$ and the appearance of a LEP}

Having demonstrated the validity of the physical interpretation of $\mathcal{L}_{H}(q)$, we address now the question of the emergence of a LEP in this model as a function of the control parameter $q$.

As pointed out in Ref [13], the example studied in this section does not present a HEP, but has a LEP. Therefore, we can study the effect of the $q$ parameter on the emergence of the EP.

For that purpose, let us first write explicitly the eigenvalues and eigenmatrices of this hybrid Liouvillian $\mathcal{L}_{\mathrm{H}}(q)$ in Eq. (26). Its eigenvalues and eigenmatrices read as follows

$$
\left\{\begin{array}{l}
\lambda_{0,3}=-\gamma_{x}(1 \mp q), \\
\lambda_{1,2}=-\gamma_{x} \pm \Omega^{\prime}
\end{array}\right.
$$

and

$$
\hat{\rho}_{1,2} \propto\left(\begin{array}{cc}
0 & -i \omega \pm \Omega^{\prime} \\
\gamma_{x} & 0
\end{array}\right)
$$

where

$$
\Omega^{\prime}=\sqrt{q^{2} \gamma_{x}^{2}-\omega^{2}},
$$

and the eigenmatrices $\hat{\rho}_{0,3}$ are the same as in (23). In Figs. 4(a) 4(e) we plot the real and in Figs. 4(f) 4(l) imaginary parts of the spectrum of $\mathcal{L}_{H}(q)$.

As Eq. (29) indicates, the EP of the hybrid Liouvillian $\mathcal{L}_{\mathrm{H}}(q)$ takes the form

$$
\gamma_{x}^{\mathrm{EP}}(q)=\omega / q
$$

Thus, for $q=0$ there is no EP (equivalently, the EP is located at infinity), as also shown in Figs. 4(a) and 4(f). Indeed, also the diagonalization of $\hat{H}_{\text {eff }}$ predicts no coalescence of eigenvalues, as indicated by Eq. (28). On the contrary, for $q=1$ there is a LEP [see also Figs. 4(e) and 4(l)]. At the EP, we can solve the Jordan chain relation for $\mathcal{L}(q)$ in Eq. (5), obtaining the generalized eigenvector $\hat{\tilde{\rho}}_{1}$ :

$$
\hat{\tilde{\rho}}_{1}=\left(\begin{array}{ll}
0 & 1 \\
0 & 0
\end{array}\right) .
$$

By introducing a small $q$, we see the emergence of an EP, but for a value of $\gamma_{x}$ which, in accordance with Eq. (30), is much larger than $\gamma_{x}^{\mathrm{EP}}=\omega$ predicted by the
Liouvillian theory [Fig. 4(b)]. As we increase $q$, however, we observe that the position and the characteristics of the EP become more and more similar to those of the LEP [c.f. Figs. 4(c) and 4(d) and Figs. 4(h) and 4(i)].

These results confirm the interpretation of the LEPs provided in Ref. [13]. Indeed, it is the backaction of a measurement apparatus on the system, induced by the quantum jumps, that generates the EP [19, 21, 24]. The projection of the system on the eigenspace of its pointer states is attenuated by the parameter $q$, thus a greater value of $\gamma_{x}$ is required to observe the EP.

We conclude that, in this example, quantum jumps are the term responsible for the EP, while the $\hat{H}_{\text {eff }}$ tends to destroy it.

\section{EXAMPLE 2 OF A MODEL WITH INEQUIVALENT LEP AND HEP}

Let us now consider a model with HEPs and LEPs (as studied in Ref. [13]), where

$$
\hat{H}=\frac{\omega}{2} \hat{\sigma}_{x}
$$

which evolves under the action of the following Liouvillian decaying channel:

$$
\mathcal{L} \hat{\rho}(t)=-i[\hat{H}, \hat{\rho}(t)]+\frac{\gamma_{-}}{2} \mathcal{D}\left[\hat{\sigma}_{-}\right] \hat{\rho}(t)
$$

The NHH

$$
\hat{H}_{\mathrm{eff}}=\frac{\omega}{2} \hat{\sigma}_{x}-i \frac{\gamma_{-}}{2} \hat{\sigma}_{+} \hat{\sigma}_{-}
$$

which results from Eq. (33) if we ignore the quantum jump term in $\mathcal{D}\left[\hat{\sigma}_{-}\right]$, has the following eigenvalues:

$$
h_{1,2}=\frac{1}{4}\left(-i \gamma_{-} \mp \zeta\right),
$$

and eigenvectors:

$$
\left|\phi_{1,2}\right\rangle \propto\left[i \gamma_{-} \mp \zeta, \quad-2 \omega\right]
$$

where $\zeta=\sqrt{4 \omega^{2}-\gamma_{-}^{2}}$. Thus, this model has a HEP for $\gamma_{-}=2 \omega$, admitting a family of generalized eigenvector depending on a single parameter $a$ :

$$
\left|\tilde{\phi}_{1}\right\rangle=[a, i(4+a)] .
$$

The Liouvillian eigenvalues are instead

$$
\begin{aligned}
\lambda_{0} & =0, \\
\lambda_{1} & =-\frac{\gamma_{-}}{2}, \\
\lambda_{2,3} & =-\frac{3}{4} \gamma_{-} \pm \beta / 4,
\end{aligned}
$$




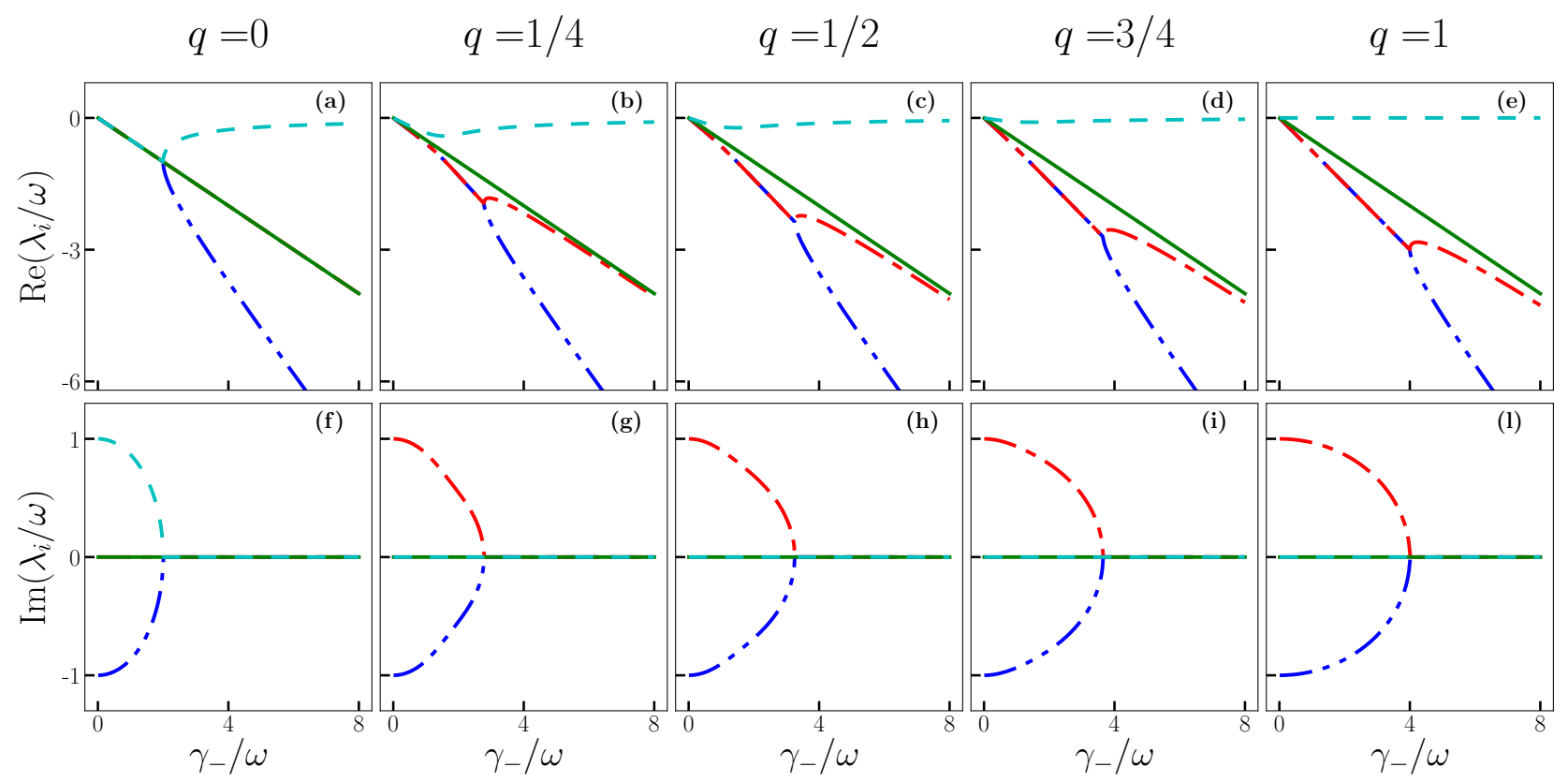

Figure 5. Spectrum of the hybrid-Liouvillian superoperator in Eq. (41) as a function of the rescaled dissipation rate $\gamma-/ \omega$. (a-e) The real part of the eigenvalues and (f-l) the imaginary one. [(a), (f)] $q=0$ (NHH case in Eq. (34)), [(b), (g)] $q=1 / 4,[(\mathrm{c}),(\mathrm{h})]$ $q=1 / 2,[(\mathrm{~d}),(\mathrm{i})] q=3 / 4$, and [(e), (l)] $q=1$ [i.e., diagonalization of the Liouvillian in Eq. (33)].

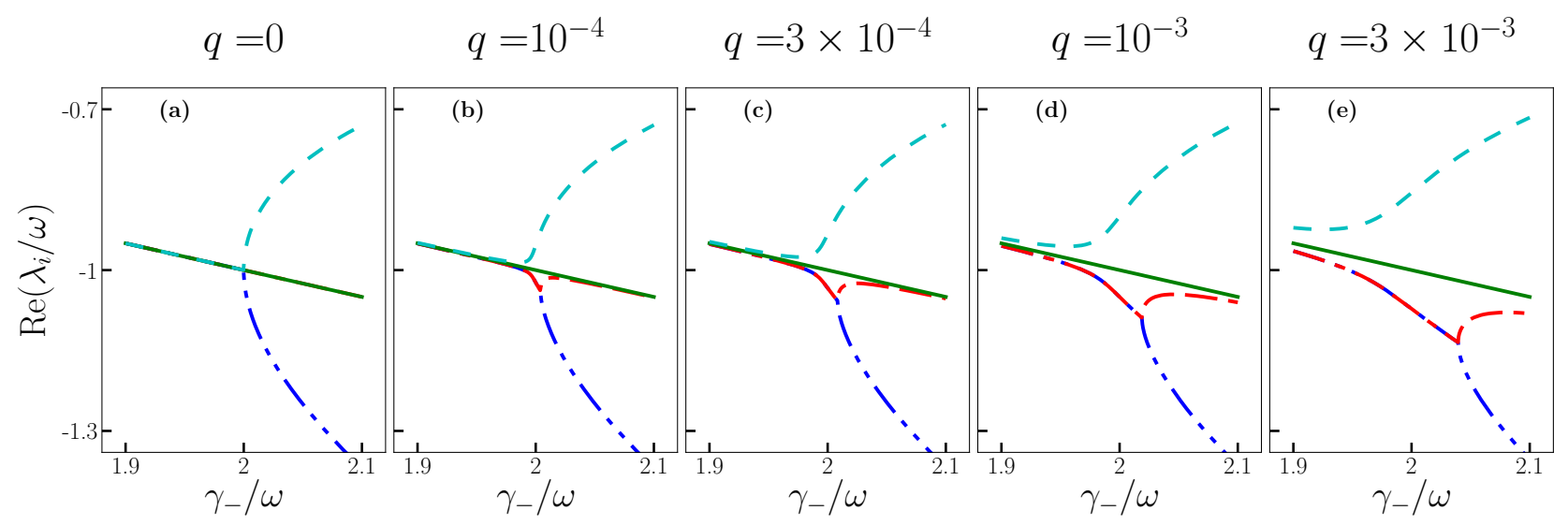

Figure 6. Real part of the spectrum of the hybrid-Liouvillian superoperator in Eq. (41) as a function of the rescaled dissipation rate $\gamma_{-} / \omega$. The panels are for values of $q \gtrsim 0$, showing how the inclusion of quantum jumps change the nature of the EP.

and the eigenmatrices are

$$
\begin{aligned}
\hat{\rho}_{0} & \propto \hat{\rho}_{\mathrm{Ss}}=\frac{1}{\gamma_{-}^{2}+2 \omega^{2}}\left(\begin{array}{cc}
\gamma_{-}^{2}+\omega^{2} & i \gamma_{-} \omega \\
-i \gamma_{-} \omega & \omega^{2}
\end{array}\right), \\
\hat{\rho}_{1} & \propto\left(\begin{array}{cc}
0 & 1 \\
1 & 0
\end{array}\right) \\
\hat{\rho}_{2,3} & \propto\left(\begin{array}{cc}
-\gamma_{-} \pm \beta & 4 i \omega \\
-4 i \omega & \gamma_{-} \mp \beta
\end{array}\right),
\end{aligned}
$$

where $\beta=\sqrt{\gamma_{-}^{2}-16 \omega^{2}}$. Hence, there is a LEP for $\gamma_{-}=4 \omega$.
The associated generalized eigenvector is:

$$
\hat{\tilde{\rho}}_{2}=4\left(\begin{array}{cc}
1 & 0 \\
0 & -1
\end{array}\right) \text {. }
$$

\section{A. Transition from the HEP to the LEP}

We study the effect of the jump parameter $q$ using the hybrid-Liouvillian

$$
\mathcal{L}=-i[\hat{H}, \cdot]+q \frac{\gamma_{-}}{2} \mathcal{D}\left[\hat{\sigma}_{-}\right] \cdot-\frac{(1-q) \gamma_{-}}{2} \frac{\hat{\sigma}_{+} \hat{\sigma}_{-} \cdot+\cdot \hat{\sigma}_{+} \hat{\sigma}_{-}}{2}
$$


The analytical computation of the spectrum of the hybrid Liouvillian in Eq. (41) becomes more involved. Both its eigenvalue $\lambda_{1}$ and the corresponding eigenmatrix $\hat{\rho}_{1}$ coincide with those given in Eqs. (38) and (39), respectively. On the other hand, the remaining three eigenvalues $\lambda_{0,2,3}$ are the solutions of a third-order polynomial. Their explicit form, along with their corresponding eigenmatrices, is given in the Appendix A.

Our analysis reveals an explicit dependence of the EP on the parameter $q$, namely,

$$
\gamma_{-}(q)=\sqrt{2} f^{-\frac{1}{2}}\left(3 f^{2}+3 q^{2}+2 f\right)^{\frac{1}{2}} \omega
$$

where

$$
f=q^{\frac{2}{3}}\left(1+\sqrt{1-q^{2}}\right)^{\frac{1}{3}}
$$

Clearly, as it follows from Eq. (42), for $q=0(q=1)$ one recovers the corresponding HEP (LEP). In this case we cannot analytically recover the form of the generalized eigenmatrix due to the presence of the $f$ terms.

We study the passage from the HEP to the LEP in Fig. 5. We plot the real [Figs.5(a)-5(e)] and imaginary [Figs. 5(f)-(l)] parts of the spectrum of $\mathcal{L}_{H}(q)$ from a standard NHH description $[q=0$ in Figs.5(a) and 5(f) $]$ to a fully Liouvillian approach $[q=1$ in Figs.5(e) and 5(1)]. By increasing $q$ one moves away from the HEP picture, recovering the spectral features resembling those of the LEP.

However, we notice that the mechanism which led to the EP is very different in the Hamiltonian and Liouvillian cases. When $q=0$, at the EP all the four different eigenvalues are coinciding. Two of the corresponding eigenmatrices are associated with those of the $\mathrm{NHH}$, while another one is doubly degenerate [see the green solid curve in Fig. 5(a)]. Thus, while from a Hamiltonian perspective there is a second-order EP [c.f. Eq. (36)], according to $\mathcal{L}_{H}(0)$ there is a third order EP. Indeed, as it stems from Figs. 5(a) and 5(f), the blue dotted-dashed curve and the light-blue dashed are those producing the EP. The presence of an EP of higher order is not surprising since $\mathcal{L}_{H}(0)$ captures also the dynamics of mixed states, which is impossible for the $\mathrm{NHH}$.

In Figs. 5(b) and 5(g) we see that, even if the blue dotted-dashed curve and the light-blue dashed curves change very little, the EP is no more associated with the coalescence of the states corresponding to those two. Indeed, the blue dotted-dashed and the red curves show the generation of the EP. This abrupt change can be interpreted as the non-analyticity associated with the passage from a EP or order 3 to one of order 2 [60]. In this regard, the effect of an imperfect post-selection can be extremely relevant in these systems.

To better grasp the effect of quantum jumps on this system, in Fig. 6 we plot the real part of the Liouvillian spectrum for $q \gtrsim 0$. As we notice from Fig. 6(b), the introduction of a very small imperfection in the photodetector changes profoundly the nature of the EP. In this limit, the states described by $\mathcal{L}_{H}(q)$ are almost identical to those described by $\mathcal{L}_{H}(0)$. Nevertheless, we may argue that in actual physical systems the introduction of this minimal noise in the photodetector counting is sufficient to distinguish between the three states which previously coincided at the EP. Two will still produce an EP for slightly shifted parameters. The other eigenmatrix, instead, is pushed away from the spectral degeneracy.

We can interpret this result as a consequence of the exceptional sensitivity of the HEP to the presence of quantum noise.

Finally, for $q \rightarrow \infty$ the EP disappears [c.f. Eqs. (A1), (A2), and (A3)]. In this sense, we can say that in this model the presence of quantum jumps is detrimental to the emergence of an EP.

\section{CONCLUSIONS}

We studied the transition between two types of EPs: semiclassical EPs (i.e., HEPs), which are degeneracies of effective non-Hermitian Hamiltonians, and truly quantum EPs (i.e., LEPs), which are degeneracies of a Liouvillian superoperator corresponding to a Lindblad master equation, as recently introduced in Ref. [13] and applied in Refs. [9, 15]. We emphasize that the inclusion of quantum jumps in the evolution of a quantum system makes, in general, LEPs fundamentally different from HEPs, as we have proved in Ref. [13].

$n$ the present paper, we have addressed the question of the relation of HEPs and LEPs based on the postselection of quantum trajectories (quantum jumps) and their classicalto-quantum correspondence. This interpretation has partially been inspired by a very recent experimental work [6] reporting quantum state tomography of a single dissipative qubit in the vicinity of its EP. This experiment was based on a post-selection on a three-level superconducting circuit.

Here, we have applied the idea of postselection to propose a hybrid-Liouvillian formalism based on a modified Liouvillian superoperator being a function of a quantumjump parameter $q$. This approach directly shows the transition of a LEP to a HEP via a proper postselection on quantum jumps (or quantum trajectories) as schematically shown Fig. 1(a). Indeed, our formalism describes in particular (i) an NHH, when one postselects only those trajectories without quantum jumps (i.e., corresponding to the quantum jump parameter $q=0$ ), and (ii) a true Liouvillian, including quantum jumps, when one does not perform postselection (i.e., when $q=1$ ). Clearly, this formalism can describe also intermediate cases for any $q \in[0,1]$, when we postselect a specific fraction of trajectories.

Moreover, our approach allowed us to interpret postselection in an operational way based on finite-efficiency detectors. Indeed, in addition to the analysis of the hybrid Liouvillian in terms of the postselection of quantum trajectories, we have also discussed its relation to inef- 
ficient photodetectors corresponding to the case when a quantum jump occurs but the detector does not signal it, as schematically shown in Fig. 1(b).

We discussed two pedagogical examples showing the application of our general hybrid-Liouvillian approach. In our first example, we analyzed a driven dissipative qubit model exhibiting a LEP but without HEPs. In our second example, we considered a qubit presenting a LEP and a HEP, which occur for a different combination of parameters. The latter example explicitly shows the transition of a LEP to a HEP as a function of the quantum-jump parameter $q$.

Note that our examples show a double effect of quantum jumps in creating and destroying EPs. Specifically, example 1 shows that a $\operatorname{LEP}\left[\mathcal{L}_{H}(q)\right.$ for $\left.q=1\right]$ can be created solely by quantum jumps, and no $\operatorname{HEP}\left[\mathcal{L}_{H}(q)\right.$ for $q=0$ ] is generated in this case. Contrary to this quantum-jump-induced EP, example 2 demonstrates a quantum-jump-destroyed EP, i.e., an NHH can create a HEP, which would disappear for $q=\infty$ (where quantum jumps would be the only process taking place). We stress that for $q=1$ a LEP is observed, but its characteristics are profoundly different from those of the HEP. This analysis on the nature of HEPs and LEPs and on the roles played by the $\hat{H}_{\text {eff }}$ and the quantum jumps could not have been performed using the standard Liouvillian or NHH alone.

The advantages of EPs for applications remain a very active topic of research [16, 61-74] and correctly modeling noise and quantum jumps is fundamental to correctly address the question of, e.g., EP sensitivity. We believe that our work, showing explicitly the operational interpretation and the relation between classical and quantum EPs in terms of postselection and/or inefficient detectors, can stimulate more interest in experimental demonstrations of LEPs and their potential quantum applications, pointing out analogies and differences with respect to those studied for semiclassical HEPs.

Concerning the hybrid Liouvillian, it could be analyzed in the context of decoherence-free subspaces, in particular in relation to non-Hermitian dynamics in the presence of dark states [75]. In this case, the effect of the postselection would be to change the eigenmatrices of the Liouvillian, leaving the eigenvalues untouched. Moreover, it would be interesting to try to generalize $\mathcal{L}(q)$ to include, e.g., noisy measurement instrument and time-dependent Hamiltonian or jump operators, or to more general types of quantum maps, such as jumptime unraveling of a quantum system[76, 77], or in connection to dynamical phase transitions [78]. Such a hybrid Liouvillian may be also be studied in the context of critical phenomena, where the $q$ parameter may change the spectral properties normally associated with multistability and metastability, multimodality, and critical slowing down [47, 48, 58, 79-82].

\section{ACKNOWLEDGMENTS}

The authors acknowledge the discussion with M. Schiró and comments of Jan Wiersig. F.M. was supported by the FY2018 JSPS Postdoctoral Fellowship for Research in Japan. A.M. and R.C. were supported by the Polish National Science Centre (NCN) under the Maestro Grant No. DEC-2019/34/A/ST2/00081. I.A. thanks the Grant Agency of the Czech Republic (Project No. 18-08874S) and the project no. CZ.02.1.01/0.0/0.0/16_019/0000754 of the Ministry of Education, Youth and Sports of the Czech Republic. F.N. is supported in part by: NTT Research, Army Research Office (ARO) (Grant No. W911NF-18-1-0358), Japan Science and Technology Agency (JST) (via the CREST Grant No. JPMJCR1676), Japan Society for the Promotion of Science (JSPS) (JSPS-RFBR Grant No. 17-52-50023, and JSPS-FWO Grant No. VS.059.18N), and the Grant No. FQXi-IAF19-06 from the Foundational Questions Institute Fund (FQXi), a donor advised fund of the Silicon Valley Community Foundation.

\section{Appendix A: Eigenvalues and eigenmatrices of the hybrid Liouvillian in Eq. (41)}

\section{Eigenvalues $\lambda_{i}$}

By solving the eigenproblem of the hybrid Liouvillian $\mathcal{L}_{\mathrm{H}}(q)$ in Eq. (41), one arrives at the following eigenvalues $\lambda_{i}$ :

$$
\begin{aligned}
\lambda_{0} & =-\frac{\gamma_{-}}{2}+2 F_{0}, \\
\lambda_{1} & =-\frac{\gamma_{-}}{2} \\
\lambda_{2,3} & =-\frac{\gamma_{-}}{2}-F_{0} \pm i \sqrt{3}\left(F_{0}-2 D\right),
\end{aligned}
$$

where

$$
F_{0}=\frac{1}{12 D}\left[D^{2}+3\left(\gamma_{-}^{2}-4 \omega^{2}\right)\right]
$$

and

$$
D=\left[54 q \gamma_{-} \omega^{2}+3 \sqrt{3} \sqrt{108 q^{2} \gamma_{-}^{2} \omega^{4}-\left(\gamma^{2}-4 \omega^{2}\right)^{3}}\right]^{\frac{1}{3}} .
$$

Importantly, due to dependence of the eigenvalues $\lambda_{i}$ on the parameter $q$ in Eq. (A1), their sorting $\left[\left|\mathbb{R e}\left[\lambda_{i}\right]\right| \leq\right.$ $\left|\mathbb{R e}\left[\lambda_{i+1}\right]\right|$,c.f. the text below Eq. (4)] is nontrivial. Thus, the indices in $\lambda_{i}$ are reshuffled as $q$ changes. Consequently, also the corresponding eigenmatrices $\hat{\rho}_{i}$, present below, will undergo the same permutation of indices.

\section{Eigenmatrices $\hat{\rho}_{i}$}

The corresponding eigenmatrices $\hat{\rho}_{i}$ of the given hybrid Liouvillian are listed below. 
Eigenmatrix $\hat{\rho}_{0} .-$ The elements of the eigenmatrix $\hat{\rho}_{0}$ read as follows

$$
\begin{aligned}
& \rho_{00}^{(0)}=\frac{-1}{6}\left(D^{3}-54 q \gamma_{-} \omega^{2}\right)\left(3 \gamma_{-}-D\right)+\frac{3 \gamma^{3}\left(\gamma_{-}-D\right)}{2}+\gamma^{2}\left[D^{2}-\omega^{2}(27 q+12)\right]+3 \gamma_{-} \omega^{2} D(3 q+2)+24 \omega^{2}-\omega^{2} D^{2}, \\
& \rho_{01}^{(0)}=i 3 \omega D^{2}\left(4 F_{0}-\gamma_{-}(2 q+1)\right), \\
& \rho_{10}^{(0)}=-\rho_{01}^{(0)}, \\
& \rho_{11}^{(0)}=6 D^{2}\left[4 \gamma_{-} q F_{0}+\omega^{2} D\right] .
\end{aligned}
$$

Eigenmatrix $\hat{\rho}_{1}$. - The elements of this eigenmatrix coincide with that $\hat{\rho}_{1}$ presented in Eq (39).

Eigenmatrix $\hat{\rho}_{2}$. - The eigenmatrix $\hat{\rho}_{2}$ has the elements:

$$
\begin{aligned}
& \rho_{00}^{(2)}=4 D^{2}\left(\gamma_{-}^{2}-\omega^{2}\right)-\frac{u_{+}\left(D^{3}-9 \gamma^{3}+36 \gamma \omega^{2}\right) D}{3}+u_{-}\left[\gamma_{-} D^{3}-16 \omega^{2} \gamma_{-}^{2}-3\left(\gamma_{-}^{2}-4 \omega^{2}\right)^{2}\right], \\
& \rho_{01}^{(2)}=-i \omega D\left[D^{2} u_{-}+6 \gamma_{-} D(2 q+1)+3 u_{+}\left(\gamma_{-}^{2}-4 \omega^{2}\right)\right], \\
& \rho_{10}^{(2)}=-\rho_{01}^{(2)}, \\
& \rho_{11}^{(2)}=-2 D\left[u_{-} q \gamma_{-} D^{2}-6 \omega^{2} D+3 u_{+} q \gamma_{-}\left(\gamma^{2}-4 \omega^{2}\right)\right],
\end{aligned}
$$

where $u_{ \pm}=1 \pm \sqrt{3}$.

Eigenmatrix $\hat{\rho}_{3}$.- The elements of the eigenmatrix $\hat{\rho}_{3}$ are the same as in Eq. (A5), except the change of the sign in the off-diagonal elements, i.e., $\rho_{01}^{(3)}=-\rho_{01}^{(2)}$ and $\rho_{10}^{(3)}=-\rho_{10}^{(2)}$.

[1] C. M. Bender and S. Boettcher, Real Spectra in NonHermitian Hamiltonians Having $\mathcal{P} \mathcal{T}$ Symmetry, Phys. Rev. Lett. 80, 5243 (1998).

[2] D. Christodoulides and J. Yang, eds., Parity-time Symmetry and Its Applications (Springer, 2018).

[3] B. Peng, Ş. K. Özdemir, S. Rotter, H. Yilmaz, M. Liertzer, F. Monifi, C. M. Bender, F. Nori, and L. Yang, Loss-induced suppression and revival of lasing, Science 346, 328 (2014).

[4] B. Peng, Ş. K. Özdemir, F. Lei, F. Monifi, M. Gianfreda, G. L. Long, S. Fan, F. Nori, C. Bender, and L. Yang, Parity-time-symmetric whispering-gallery microcavities, Nature Physics 10, 394 (2014).

[5] T. Gao, E. Estrecho, K. Y. Bliokh, T. C. H. Liew, M. D. Fraser, S. Brodbeck, M. Kamp, C. Schneider, S. Höfling, Y. Yamamoto, F. Nori, Y. S. Kivshar, A. G. Truscott, R. G. Dall, and E. A. Ostrovskaya, Observation of nonHermitian degeneracies in a chaotic exciton-polariton billiard, Nature (London) 526, 554 (2015).

[6] M. Naghiloo, M. Abbasi, Y. N. Joglekar, and K. W. Murch, Quantum state tomography across the exceptional point in a single dissipative qubit, Nature Physics 15, 1232 (2019).

[7] H. Jing, Ş. K. Özdemir, X.-Y. Lü, J. Zhang, L. Yang, and F. Nori, $\mathcal{P} \mathcal{T}$-Symmetric Phonon Laser, Phys. Rev. Lett. 113, 053604 (2014).

[8] J. Zhang, B. Peng, Ş. K. Özdemir, K. Pichler, D. O. Krimer, G. Zhao, F. Nori, Y.-x. Liu, S. Rotter, and L. Yang, A phonon laser operating at an exceptional point, Nature Photonics 12, 479 (2018).

[9] R. Huang, Ş. K. Özdemir, J. Q. Liao, F. Minganti, L. M. Kuang, F. Nori, and H. Jing, Exceptional Photon Blockade (2020), arXiv:2001.09492.
[10] Ş. K. Özdemir, S. Rotter, F. Nori, and L. Yang, Parity-time symmetry and exceptional points in photonics, Nature Materials 18, 783 (2019).

[11] M.-A. Miri and A. Alù, Exceptional points in optics and photonics, Science 363, eaar7709 (2019).

[12] N. Hatano, Exceptional points of the Lindblad operator of a two-level system, Mol. Phys. 117, 2121 (2019).

[13] F. Minganti, A. Miranowicz, R. W. Chhajlany, and F. Nori, Quantum exceptional points of non-Hermitian Hamiltonians and Liouvillians: The effects of quantum jumps, Phys. Rev. A 100, 062131 (2019).

[14] J. Peřina, A. Lukš, J. K. Kalaga, W. Leoński, and A. Miranowicz, Nonclassical light at exceptional points of a quantum $\mathcal{P} \mathcal{T}$-symmetric two-mode system, Phys. Rev. A 100, 053820 (2019).

[15] I. I. Arkhipov, A. Miranowicz, F. Minganti, and F. Nori, Quantum and semiclassical exceptional points of a linear system of coupled cavities with losses and gain within the Scully-Lamb laser theory, Phys. Rev. A 101, 013812 (2020).

[16] P.-C. Kuo, N. Lambert, A. Miranowicz, H.-B. Chen, G.-Y. Chen, Y.-N. Chen, and F. Nori, Collectively induced exceptional points of quantum emitters coupled to nanoparticle surface plasmons, Phys. Rev. A 101, 013814 (2020).

[17] B. Jaramillo Ávila, C. Ventura-Velázquez, R. d. J. LeónMontiel, Y. N. Joglekar, and B. M. Rodríguez-Lara, $\mathcal{P} \mathcal{T}$ -symmetry from Lindblad dynamics in a linearized optomechanical system, Scientific Reports 10, 1761 (2020).

[18] C. Gardiner and P. Zoller, Quantum Noise: A Handbook of Markovian and Non-Markovian Quantum Stochastic Methods with Applications to Quantum Optics (Springer, Berlin, 2004). 
[19] S. Haroche and J. M. Raimond, Exploring the Quantum: Atoms, Cavities, and Photons (Oxford University Press, Oxford, 2006).

[20] H. J. Carmichael, Statistical Methods in Quantum Optics 1: Master Equations and Fokker-Planck Equations (Springer, Berlin, 1999).

[21] H. Wiseman and G. Milburn, Quantum Measurement and Control (Cambridge University Press, Cambridge, 2010).

[22] H. Breuer and F. Petruccione, The Theory of Open Quantum Systems (Oxford University Press, Oxford, 2007).

[23] D. A. Lidar, Lecture Notes on the Theory of Open Quantum Systems, arXiv:1902.00967.

[24] S. Barnett, Quantum Information (Oxford University Press, Oxford, 2009).

[25] M. G. A. Paris, The modern tools of quantum mechanics, EPJ - Special Topics 203, 61 (2012).

[26] K. Mølmer, Y. Castin, and J. Dalibard, Monte Carlo wave-function method in quantum optics, J. Opt. Soc. Am. B 10, 524 (1993).

[27] H. Carmichael, Statistical Methods in Quantum Optics 2: Non-Classical Fields (Springer, Berlin, 2007).

[28] A. J. Daley, Quantum trajectories and open many-body quantum systems, Advances in Physics 63, 77 (2014).

[29] W. Nagourney, J. Sandberg, and H. Dehmelt, Shelved optical electron amplifier: Observation of quantum jumps, Phys. Rev. Lett. 56, 2797 (1986).

[30] T. Sauter, W. Neuhauser, R. Blatt, and P. E. Toschek, Observation of Quantum Jumps, Phys. Rev. Lett. 57, 1696 (1986).

[31] J. C. Bergquist, R. G. Hulet, W. M. Itano, and D. J. Wineland, Observation of Quantum Jumps in a Single Atom, Phys. Rev. Lett. 57, 1699 (1986).

[32] S. Peil and G. Gabrielse, Observing the Quantum Limit of an Electron Cyclotron: QND Measurements of Quantum Jumps between Fock States, Phys. Rev. Lett. 83, 1287 (1999).

[33] T. Basché, S. Kummer, and C. Bräuchle, Direct spectroscopic observation of quantum jumps of a single molecule, Nature (London) 373, 132 (1995).

[34] S. Gleyzes, S. Kuhr, C. Guerlin, J. Bernu, S. Deléglise, U. Busk Hoff, M. Brune, J.-M. Raimond, and S. Haroche, Quantum jumps of light recording the birth and death of a photon in a cavity, Nature (London) 446, 297 (2007).

[35] C. Guerlin, J. Bernu, S. Deléglise, C. Sayrin, S. Gleyzes, S. Kuhr, M. Brune, J.-M. Raimond, and S. Haroche, Progressive field-state collapse and quantum non-demolition photon counting, Nature (London) 448, 889 (2007).

[36] S. Deléglise, I. Dotsenko, C. Sayrin, J. Bernu, M. Brune, J.-M. Raimond, and S. Haroche, Reconstruction of nonclassical cavity field states with snapshots of their decoherence, Nature (London) 455, 510 (2008).

[37] C. Sayrin, I. Dotsenko, X. Zhou, B. Peaudecerf, T. Rybarczyk, S. Gleyzes, P. Rouchon, M. Mirrahimi, H. Amini, M. Brune, J.-M. Raimond, and S. Haroche, Real-time quantum feedback prepares and stabilizes photon number states, Nature (London) 477, 73 (2011).

[38] F. Jelezko, I. Popa, A. Gruber, C. Tietz, J. Wrachtrup, A. Nizovtsev, and S. Kilin, Single spin states in a defect center resolved by optical spectroscopy, Appl. Phys. Lett. 81, 2160 (2002).

[39] P. Neumann, J. Beck, M. Steiner, F. Rempp, H. Fedder, P. R. Hemmer, J. Wrachtrup, and F. Jelezko, SingleShot Readout of a Single Nuclear Spin, Science 329, 542 (2010).
[40] L. Robledo, L. Childress, H. Bernien, B. Hensen, P. F. A. Alkemade, and R. Hanson, High-fidelity projective readout of a solid-state spin quantum register, Nature (London) 477, 574 (2011).

[41] R. Vijay, D. H. Slichter, and I. Siddiqi, Observation of Quantum Jumps in a Superconducting Artificial Atom, Phys. Rev. Lett. 106, 110502 (2011).

[42] M. Hatridge, S. Shankar, M. Mirrahimi, F. Schackert, K. Geerlings, T. Brecht, K. M. Sliwa, B. Abdo, L. Frunzio, S. M. Girvin, R. J. Schoelkopf, and M. H. Devoret, Quantum Back-Action of an Individual Variable-Strength Measurement, Science 339, 178 (2013).

[43] L. Sun, A. Petrenko, Z. Leghtas, B. Vlastakis, G. Kirchmair, K. M. Sliwa, A. Narla, M. Hatridge, S. Shankar, J. Blumoff, L. Frunzio, M. Mirrahimi, M. H. Devoret, and R. J. Schoelkopf, Tracking photon jumps with repeated quantum non-demolition parity measurements, Nature (London) 511, 444 (2014).

[44] N. Ofek, A. Petrenko, R. Heeres, P. Reinhold, Z. Leghtas, B. Vlastakis, Y. Liu, L. Frunzio, S. M. Girvin, L. Jiang, M. Mirrahimi, M. H. Devoret, and R. J. Schoelkopf, Extending the lifetime of a quantum bit with error correction in superconducting circuits, Nature (London) 536, 441 (2016).

[45] Z. K. Minev, S. O. Mundhada, S. Shankar, P. Reinhold, R. Gutiérrez-Jáuregui, R. J. Schoelkopf, M. Mirrahimi, H. J. Carmichael, and M. H. Devoret, To catch and reverse a quantum jump mid-flight, Nature (London) 570, 200 (2019).

[46] V. V. Albert and L. Jiang, Symmetries and conserved quantities in Lindblad master equations, Phys. Rev. A 89, 022118 (2014).

[47] F. Minganti, A. Biella, N. Bartolo, and C. Ciuti, Spectral theory of Liouvillians for dissipative phase transitions, Phys. Rev. A 98, 042118 (2018).

[48] K. Macieszczak, M. Gută, I. Lesanovsky, and J. P. Garrahan, Towards a Theory of Metastability in Open Quantum Dynamics, Phys. Rev. Lett. 116, 240404 (2016).

[49] M. S. Sarandy and D. A. Lidar, Adiabatic approximation in open quantum systems, Phys. Rev. A 71, 012331 (2005).

[50] T. Prosen, Spectral theorem for the Lindblad equation for quadratic open fermionic systems, J. Stat. Mech.: Theory Exp. 2010, P07020 (2010).

[51] T. Mathisen and J. Larson, Liouvillian of the Open STIRAP Problem, Entropy 20 (2018), 10.3390/e20010020.

[52] M. van Caspel, S. E. T. Arze, and I. P. Castillo, Dynamical signatures of topological order in the driven-dissipative Kitaev chain, SciPost Phys. 6, 26 (2019).

[53] H. J. Carmichael, Quantum trajectory theory for cascaded open systems, Phys. Rev. Lett. 70, 2273 (1993).

[54] J. Dalibard, Y. Castin, and K. Mølmer, Wave-function approach to dissipative processes in quantum optics, Phys. Rev. Lett. 68, 580 (1992).

[55] J. Johansson, P. Nation, and F. Nori, QuTiP: An opensource Python framework for the dynamics of open quantum systems, Comp. Phys. Commun. 183, 1760 (2012).

[56] J. Johansson, P. Nation, and F. Nori, QuTiP 2: A Python framework for the dynamics of open quantum systems, Comp. Phys. Commun. 184, 1234 (2013).

[57] N. Bartolo, F. Minganti, J. Lolli, and C. Ciuti, Homodyne versus photon-counting quantum trajectories for dissipative Kerr resonators with two-photon driving, European Physical Journal Special Topics 226, 2705 (2017). 
[58] R. Rota, F. Minganti, A. Biella, and C. Ciuti, Dynamical properties of dissipative XYZ Heisenberg lattices, New Journal of Physics 20, 045003 (2018).

[59] W. H. Zurek, Decoherence, einselection, and the quantum origins of the classical, Rev. Mod. Phys. 75, 715 (2003).

[60] J. Wiersig, Robustness of exceptional point-based sensors against parametric noise: The role of Hamiltonian and Liouvillian degeneracies, (2020), arXiv:2003.02222.

[61] J. Wiersig, Enhancing the Sensitivity of Frequency and Energy Splitting Detection by Using Exceptional Points: Application to Microcavity Sensors for Single-Particle Detection, Phys. Rev. Lett. 112, 203901 (2014).

[62] N. Zhang, S. Liu, K. Wang, Z. Gu, M. Li, N. Yi, S. Xiao, and Q. Song, Single Nanoparticle Detection Using Farfield Emission of Photonic Molecule around the Exceptional Point, Scientific Reports 5, 11912 (2015).

[63] J. Wiersig, Sensors operating at exceptional points: General theory, Phys. Rev. A 93, 033809 (2016).

[64] J. Ren, H. Hodaei, G. Harari, A. U. Hassan, W. Chow, M. Soltani, D. Christodoulides, and M. Khajavikhan, Ultrasensitive micro-scale parity-time-symmetric ring laser gyroscope, Optics Letters 42, 1556 (2017).

[65] W. Chen, Ş. K. Özdemir, G. Zhao, J. Wiersig, and L. Yang, Exceptional points enhance sensing in an optical microcavity, Nature (London) 548, 192 (2017).

[66] H. Hodaei, A. U. Hassan, S. Wittek, H. Garcia-Gracia, R. El-Ganainy, D. N. Christodoulides, and M. Khajavikhan, Enhanced sensitivity at higher-order exceptional points, Nature (London) 548, 187 (2017).

[67] P.-Y. Chen, M. Sakhdari, M. Hajizadegan, Q. Cui, M. M.-C. Cheng, R. El-Ganainy, and A. Alù, Generalized parity-time symmetry condition for enhanced sensor telemetry, Nature Electronics 1, 297 (2018).

[68] Z.-P. Liu, J. Zhang, Ş. K. Özdemir, B. Peng, H. Jing, X.-Y. Lü, C.-W. Li, L. Yang, F. Nori, and Y.-x. Liu, Metrology with $\mathcal{P} \mathcal{T}$-Symmetric Cavities: Enhanced Sensitivity near the $\mathcal{P} \mathcal{T}$-Phase Transition, Phys. Rev. Lett. 117, 110802 (2016).

[69] W. Langbein, No exceptional precision of exceptionalpoint sensors, Phys. Rev. A 98, 023805 (2018).

[70] H.-K. Lau and A. A. Clerk, Fundamental limits and nonreciprocal approaches in non-Hermitian quantum sensing, Nat. Commun. 9, 4320 (2018).

[71] N. A. Mortensen, P. A. D. Gonçalves, M. Khajavikhan, D. N. Christodoulides, C. Tserkezis, and C. Wolff, Fluctuations and noise-limited sensing near the exceptional point of parity-time-symmetric resonator systems, Optica 5, 1342 (2018).

[72] C. Wolff, C. Tserkezis, and N. A. Mortensen, On the time evolution at a fluctuating exceptional point, Nanophotonics 0 (2019).

[73] M. Zhang, W. Sweeney, C. W. Hsu, L. Yang, A. D. Stone, and L. Jiang, Quantum Noise Theory of Exceptional Point Amplifying Sensors, arXiv:1805.12001.

[74] C. Chen, L. Jin, and R.-B. Liu, Sensitivity of parameter estimation near the exceptional point of a non-Hermitian system, New J. Phys. 21, 083002 (2019).

[75] V. V. Albert, Lindbladians with multiple steady states: theory and applications, (2018), arXiv:1802.00010.

[76] J. P. Garrahan, R. L. Jack, V. Lecomte, E. Pitard, K. van Duijvendijk, and F. van Wijland, First-order dynamical phase transition in models of glasses: an approach based on ensembles of histories, Journal of Physics A: Mathematical and Theoretical 42, 075007 (2009).
[77] C. Gneiting, A. V. Rozhkov, and F. Nori, Jumptime unraveling of Markovian open quantum systems (2020), arXiv:2001.08929.

[78] K. Macieszczak, M. Guţă, I. Lesanovsky, and J. P. Garrahan, Dynamical phase transitions as a resource for quantum enhanced metrology, Phys. Rev. A 93, 022103 (2016).

[79] F. Vicentini, F. Minganti, R. Rota, G. Orso, and C. Ciuti, Critical slowing down in driven-dissipative Bose-Hubbard lattices, Phys. Rev. A 97, 013853 (2018).

[80] J. Jin, A. Biella, O. Viyuela, C. Ciuti, R. Fazio, and D. Rossini, Phase diagram of the dissipative quantum Ising model on a square lattice, Phys. Rev. B 98, 241108(R) (2018).

[81] H. Landa, M. Schiró, and G. Misguich, Multistability of Driven-Dissipative Quantum Spins, Phys. Rev. Lett. 124, 043601 (2020).

[82] D. C. Rose, K. Macieszczak, I. Lesanovsky, and J. P. Garrahan, Metastability in an open quantum Ising model, Phys. Rev. E 94, 052132 (2016). 